University of Tennessee Health Science Center UTHSC Digital Commons

$12-2016$

\title{
The Effect of Hamstring Lengthening on Pelvic Tilt and Lumbar Lordosis
}

\author{
Michael Scott Braman \\ University of Tennessee Health Science Center
}

Follow this and additional works at: https://dc.uthsc.edu/dissertations

Part of the Therapeutics Commons

\section{Recommended Citation}

Braman, Michael Scott (http://orcid.org/0000-0003-2350-8205), "The Effect of Hamstring Lengthening on Pelvic Tilt and Lumbar Lordosis" (2016). Theses and Dissertations (ETD). Paper 407. http://dx.doi.org/ 10.21007/etd.cghs.2016.0578.

This Thesis is brought to you for free and open access by the College of Graduate Health Sciences at UTHSC Digital Commons. It has been accepted for inclusion in Theses and Dissertations (ETD) by an authorized administrator of UTHSC Digital Commons. For more information, please contact jwelch30@uthsc.edu. 


\title{
The Effect of Hamstring Lengthening on Pelvic Tilt and Lumbar Lordosis
}

\begin{abstract}
A direct correlation between hamstring tightness and severity of lower back pain (LBP) has been previously reported. Hamstring contraction creates knee flexion, hip extension and posterior pelvic tilt. Posterior pelvic tilt causes the lumbar spine to flatten (hypolordosis) which places pressure on the anterior structures of the spine, including the intervertebral disc. This increased pressure may predispose individuals to disc degeneration and associated pain. For this reason, hamstring stretching is often prescribed to alleviate LBP, however, the effect of hamstring lengthening on the kinematics of the lumbar spine and pelvis is not well understood. Accurate measurement of lumbar spine movement is difficult to obtain in a motion capture laboratory due to subtle differences in marker placement, tissue interface and distribution. Therefore, the purpose of this study was twofold. The first goal was to develop and validate a method that allows accurate comparison of LL measurements from different testing sessions. Secondly, we aimed to assess the effect of hamstring lengthening on anterior pelvic tilt (PT) and lumbar lordosis (LL) in healthy subjects during gait, sit to stand, forward bend, and picking up a ball from the floor. Two different methods were developed and tested to determine which provided the most accurate LL measure from motion lab data. First, the LL correction method was developed and tested by using EOS bi-planar XRays of twenty-one healthy subjects. Two scans were taken of each subject; the first standing normally, and the second wearing a spine altering orthotic. Anatomical LL measurements were taken from the sagittal radiograph and the SterEOS reconstructions of both scans. Marker based LL was also taken from the sagittal radiograph. The first method computed the ratio of the anatomical based measurement to marker based measurement to give a correction factor (CF). The second method computed the difference between the anatomical measurement and the marker based measurement to give a correction constant (CC). The CF and CC were then applied to marker based measurements from the second scan to give a corrected LL. The correction constant resulted in the lowest average error in corrected LL. The average error for this method was $3.2^{\circ} \pm 2.8^{\circ}$ (Mean $\pm S D$ ) which corresponding to a percent error of $6.2 \% \pm 4.8 \%$. This was found to have less error than the error introduced by inconsistent marker placement between trials, although the use of it is limited to activities with minimal lumbar flexion. Next, nine healthy individuals with tight hamstrings (popliteal angle greater than $25^{\circ}$ ) were recruited for this study. A physical therapist placed 58 reflective markers by palpation on anatomical landmarks of the torso and lower extremities. Subjects performed walking, sit to stand, forward bend, and ball pick up from the floor, while ten optoelectronic cameras recorded the 3D location of the reflective markers. Subjects were then scanned with the spine markers using an EOS bi-planar X-ray system. Following testing, participants completed a six week stretching program designed to increase hamstring length. All baseline testing was then repeated. PT was defined as the angle between horizontal and the line extending from the posterior superior iliac spine marker to the anterior superior iliac spine marker. LL was defined as the acute angle between the lines connecting the markers on T12 to L2 and S2 to L4. The LL correction constant was applied to walking and standing activities to give corrected $L L$ values. The change in PT and corrected $L L$ was then found for each participant. All subjects had a decrease in popliteal angle $\left(-18.8^{\circ} \pm 11.6^{\circ}\right)$. It was found that hamstring lengthening increased PT $2.1^{\circ} \pm 2.6^{\circ}$ at heal strike during walking $(p=0.04)$. The greatest increase of $10.6^{\circ} \pm 5.4^{\circ}(p<0.001)$ occurred at flexion during forward bend. Ball pick up also saw a similar increase at flexion and knee bend. PT at standing increased $4.4^{\circ} \pm 2.8^{\circ}(p=0.001)$ and $2.0^{\circ} \pm 2.1^{\circ}$ $(p=0.05)$ during forward bend and standing respectively. While increased PT tended to correspond to increased $L L$, the increased LL was only statistically significant while standing during the forward bend activity $\left(7.0^{\circ} \pm 7.2^{\circ}\right)$. Hamstring stretching was effective for increasing anterior pelvic tilt during gait, forward bending tasks, and standing. The effect that increased PT has on LL is still a topic for further investigation. Future studies should include increased sample size and improvement of the LL correction method.
\end{abstract}




\section{Document Type}

Thesis

\section{Degree Name}

Master of Science (MS)

\section{Program}

Biomedical Engineering

\section{Research Advisor}

William M. Mihalko

\section{Keywords}

Gait, Low Back Pain, Lumbar Lordosis, Pelvic Tilt, Spine Kinematics, Tight Hamstrings

\section{Subject Categories}

Analytical, Diagnostic and Therapeutic Techniques and Equipment | Medicine and Health Sciences |

Therapeutics 
The Effect of Hamstring Lengthening on Pelvic Tilt and Lumbar Lordosis

\author{
A Thesis \\ Presented for \\ The Graduate Studies Council \\ The University of Tennessee \\ Health Science Center
}

\begin{abstract}
In Partial Fulfillment
Of the Requirements for the Degree

Master of Science in Biomedical Engineering

In the Joint Graduate Program in Biomedical Engineering and Imaging

From The University of Tennessee

and

The University of Memphis
\end{abstract}

By

Michael Scott Braman

December 2016 
Copyright (C) 2016 by Michael Scott Braman.

All rights reserved. 


\section{DEDICATION}

This thesis is dedicated to my wife, Danielle Braman, who not only inspired me to go back to graduate school, but also gave endless love and support along the way. I also dedicate this work to my parents, Mark \& Sheila Braman, for giving their unconditional support for all of my endeavors. 


\section{ACKNOWLEDGEMENTS}

I would like to thank my advisor, Dr. William M. Mihalko for granting me this opportunity and giving me guidance throughout. I would also like to thank my committee members, Dr. Audrey Zucker-Levin, Dr. Brooke Sanford, and Dr. Kunal Singhal for their guidance and support. I would like to give special thanks to Dr. ZuckerLevin and Pablo Dopico for the many hours spent testing in the gait lab.

I would also like to acknowledge my fellow graduate students, including Pablo Dopico, Julie Lowell, Kylie Davis, Michael Parker, Chloe Chung, Cody Bateman, and Clay Hillyard, for helping with various matters along the way and making my graduate experience more enjoyable. 


\begin{abstract}
A direct correlation between hamstring tightness and severity of lower back pain (LBP) has been previously reported. Hamstring contraction creates knee flexion, hip extension and posterior pelvic tilt. Posterior pelvic tilt causes the lumbar spine to flatten (hypolordosis) which places pressure on the anterior structures of the spine, including the intervertebral disc. This increased pressure may predispose individuals to disc degeneration and associated pain. For this reason, hamstring stretching is often prescribed to alleviate LBP, however, the effect of hamstring lengthening on the kinematics of the lumbar spine and pelvis is not well understood. Accurate measurement of lumbar spine movement is difficult to obtain in a motion capture laboratory due to subtle differences in marker placement, tissue interface and distribution. Therefore, the purpose of this study was twofold. The first goal was to develop and validate a method that allows accurate comparison of LL measurements from different testing sessions. Secondly, we aimed to assess the effect of hamstring lengthening on anterior pelvic tilt (PT) and lumbar lordosis (LL) in healthy subjects during gait, sit to stand, forward bend, and picking up a ball from the floor.
\end{abstract}

Two different methods were developed and tested to determine which provided the most accurate LL measure from motion lab data. First, the LL correction method was developed and tested by using EOS bi-planar X-Rays of twenty-one healthy subjects. Two scans were taken of each subject; the first standing normally, and the second wearing a spine altering orthotic. Anatomical LL measurements were taken from the sagittal radiograph and the SterEOS reconstructions of both scans. Marker based LL was also taken from the sagittal radiograph. The first method computed the ratio of the anatomical based measurement to marker based measurement to give a correction factor $(\mathrm{CF})$. The second method computed the difference between the anatomical measurement and the marker based measurement to give a correction constant (CC). The $\mathrm{CF}$ and $\mathrm{CC}$ were then applied to marker based measurements from the second scan to give a corrected LL. The correction constant resulted in the lowest average error in corrected LL. The average error for this method was $3.2^{\circ} \pm 2.8^{\circ}$ (Mean \pm SD) which corresponding to a percent error of $6.2 \% \pm 4.8 \%$. This was found to have less error than the error introduced by inconsistent marker placement between trials, although the use of it is limited to activities with minimal lumbar flexion.

Next, nine healthy individuals with tight hamstrings (popliteal angle greater than $25^{\circ}$ ) were recruited for this study. A physical therapist placed 58 reflective markers by palpation on anatomical landmarks of the torso and lower extremities. Subjects performed walking, sit to stand, forward bend, and ball pick up from the floor, while ten optoelectronic cameras recorded the $3 \mathrm{D}$ location of the reflective markers. Subjects were then scanned with the spine markers using an EOS bi-planar X-ray system. Following testing, participants completed a six week stretching program designed to increase hamstring length. All baseline testing was then repeated. PT was defined as the angle between horizontal and the line extending from the posterior superior iliac spine marker to the anterior superior iliac spine marker. LL was defined as the acute angle between the 
lines connecting the markers on T12 to L2 and S2 to L4. The LL correction constant was applied to walking and standing activities to give corrected LL values. The change in PT and corrected LL was then found for each participant.

All subjects had a decrease in popliteal angle $\left(-18.8^{\circ} \pm 11.6^{\circ}\right)$. It was found that hamstring lengthening increased PT $2.1^{\circ} \pm 2.6^{\circ}$ at heal strike during walking $(\mathrm{p}=0.04)$. The greatest increase of $10.6^{\circ} \pm 5.4^{\circ}(\mathrm{p}<0.001)$ occurred at flexion during forward bend. Ball pick up also saw a similar increase at flexion and knee bend. PT at standing increased $4.4^{\circ} \pm 2.8^{\circ}(\mathrm{p}=0.001)$ and $2.0^{\circ} \pm 2.1^{\circ}(\mathrm{p}=0.05)$ during forward bend and standing respectively. While increased PT tended to correspond to increased LL, the increased LL was only statistically significant while standing during the forward bend activity $\left(7.0^{\circ} \pm 7.2^{\circ}\right)$.

Hamstring stretching was effective for increasing anterior pelvic tilt during gait, forward bending tasks, and standing. The effect that increased PT has on LL is still a topic for further investigation. Future studies should include increased sample size and improvement of the LL correction method. 


\section{TABLE OF CONTENTS}

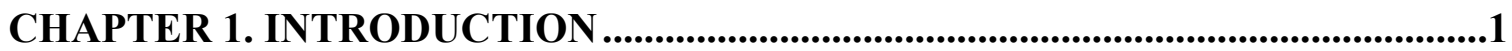

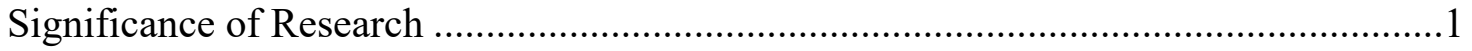

Thesis Aim and Outline .....................................................................................

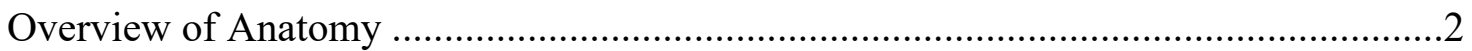

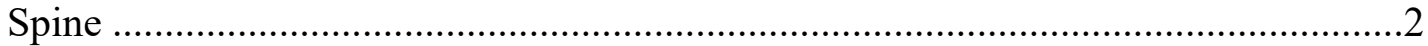

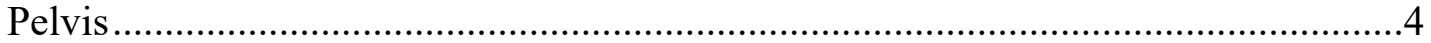

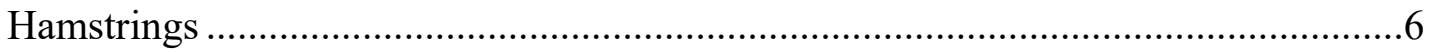

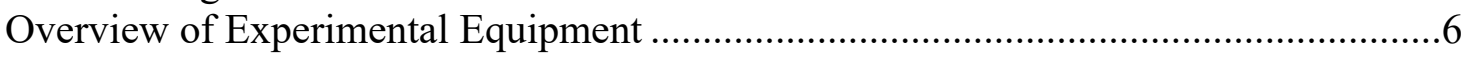

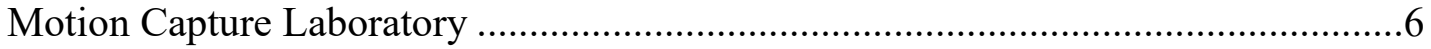

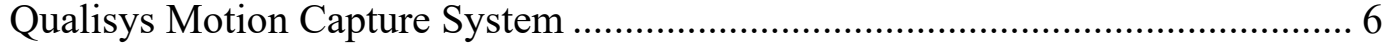

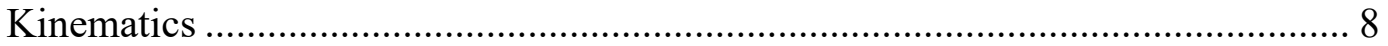

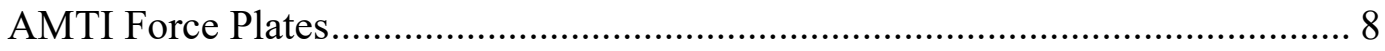

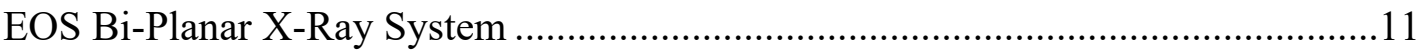

\section{CHAPTER 2. A NOVEL METHOD FOR CORRECTING MOTION LAB} LUMBAR LORDOSIS MEASUREMENTS UTILIZING AN EOS BI-PLANAR

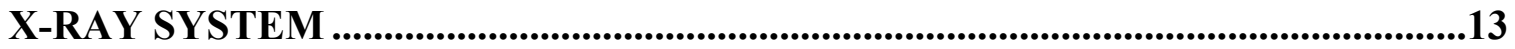

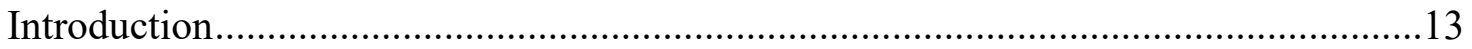

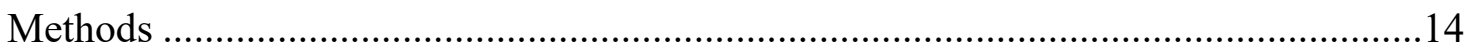

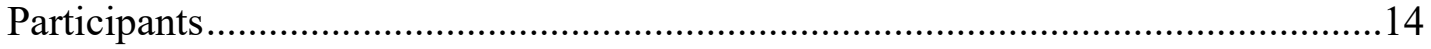

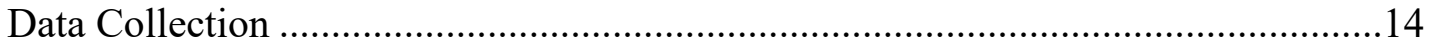

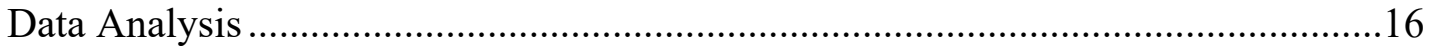

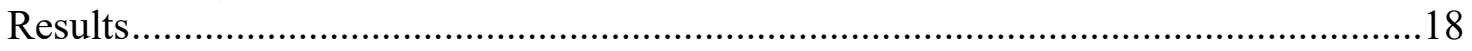

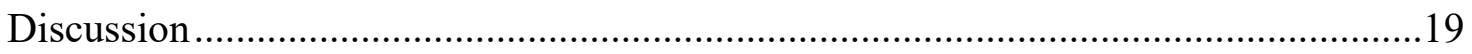

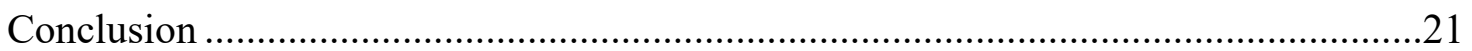

\section{CHAPTER 3. EFFECT OF HAMSTRING LENGTHENING ON PELVIC TILT}

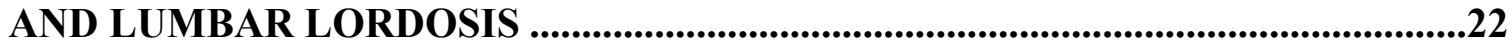

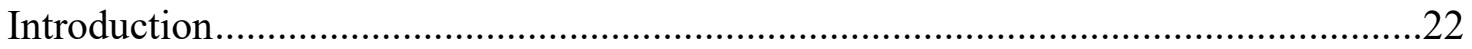

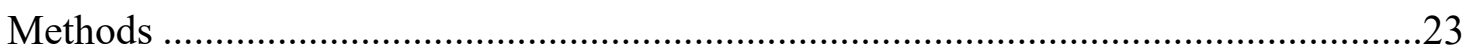

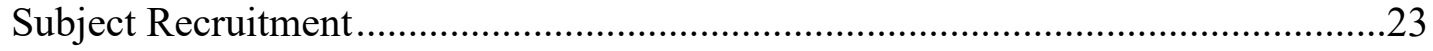

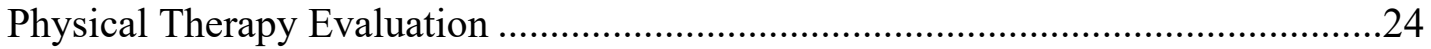

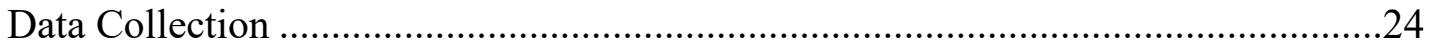

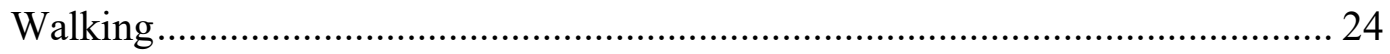

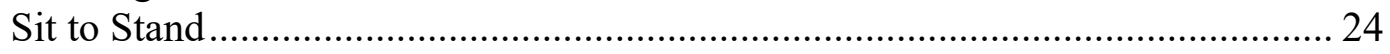

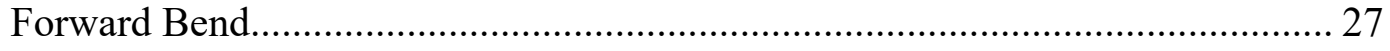

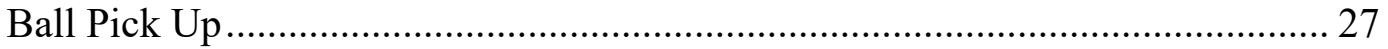

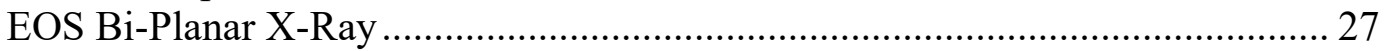

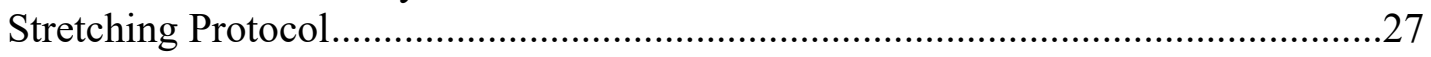

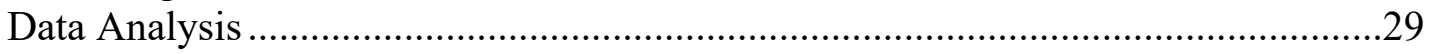

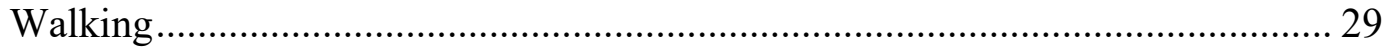

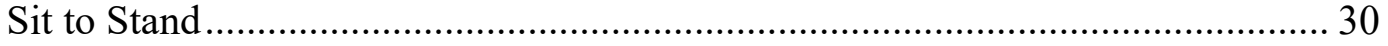

Forward Bend....................................................................................... 30 
Ball Pick Up ............................................................................................ 30

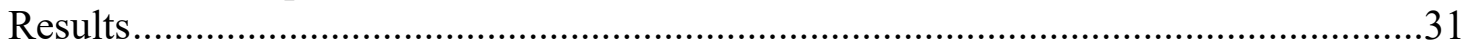

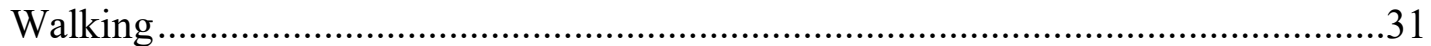

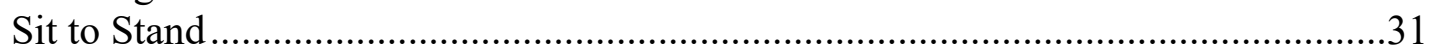

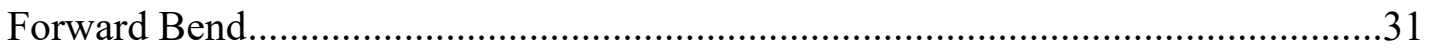

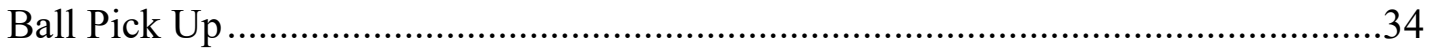

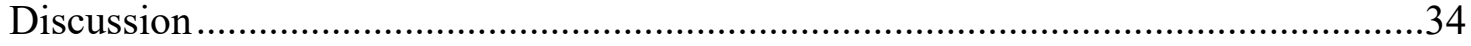

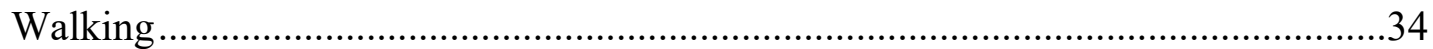

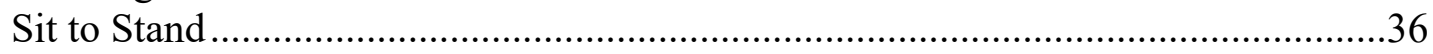

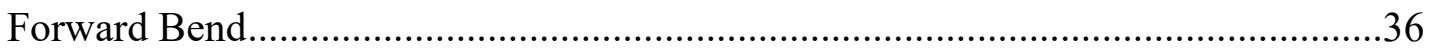

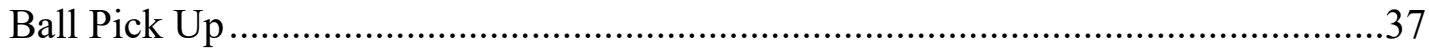

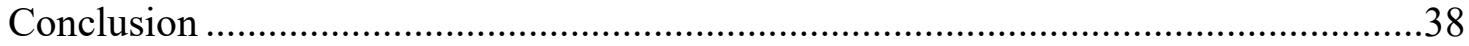

CHAPTER 4. GENERAL DISCUSSION .............................................................40

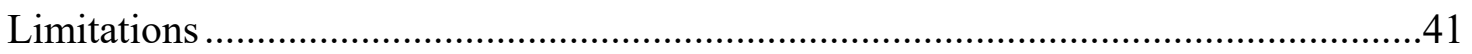

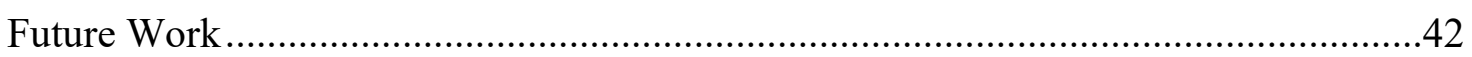

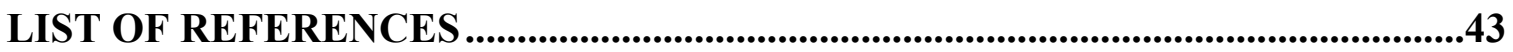

APPENDIX A. INSTITUTIONAL REVIEW BOARD APPROVAL .....................46

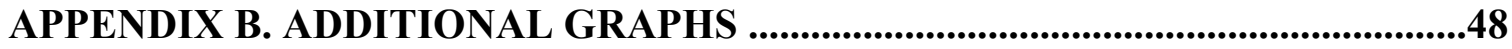

VITA 


\section{LIST OF TABLES}

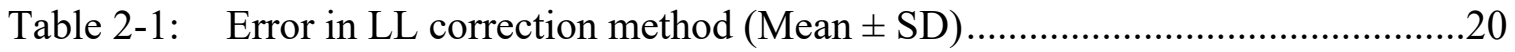

Table 3-1: $\quad$ Summary of subject demographics (Mean \pm SD) .....................................25

Table 3-2: Change in popliteal angle (PA), pelvic tilt (PT), corrected lumbar lordosis (LL) and stride length (SL) during gait ......................................32

Table 3-3: $\quad$ Change in PT and corrected LL for each participant during sit-to-stand .....32

Table 3-4: $\quad$ Change in PT, corrected LL, and C7 height during forward bend ...............33

Table 3-5: Change in PT and corrected LL during ball pick up.................................35

Table 3-6: Change in pelvic flexion and lumbar flexion during ball pick up ...............35 


\section{LIST OF FIGURES}

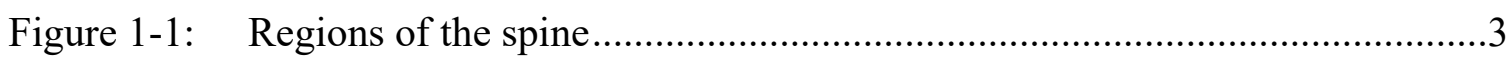

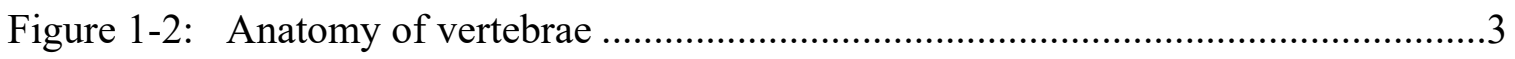

Figure 1-3: Anatomy of the intervertebral disc ....................................................

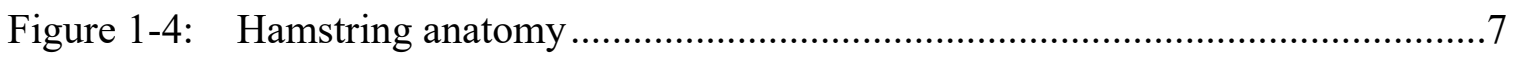

Figure 1-5: The effect of hamstring tightness on PT and LL ....................................

Figure 1-6: QTM marker set and Visual 3D model ................................................

Figure 1-7: Eight segment model applied to static trial in Visual 3D ............................

Figure 1-8: Pelvic tilt and lumbar lordosis angle measurements ...............................10

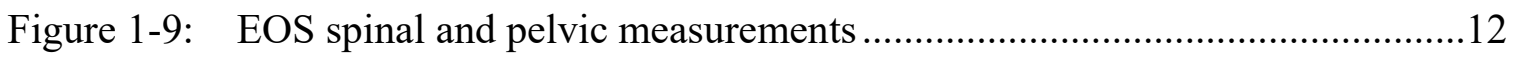

Figure 2-1: Subject with reflective markers on torso and lower extremities.................15

Figure 2-2: Clinical LL and marker based LL on radiograph.....................................17

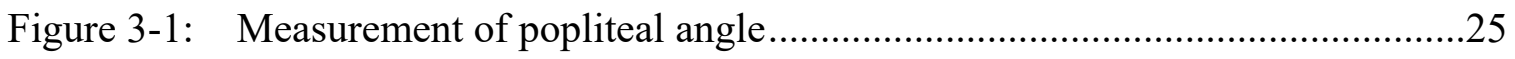

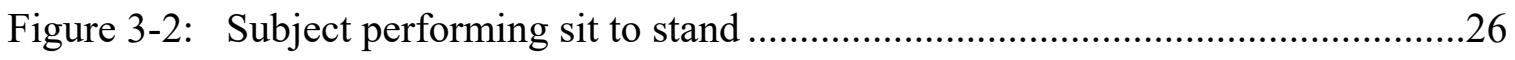

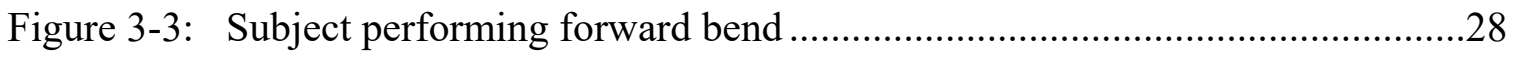

Figure 3-4: Subject performing ball pick up.......................................................28

Figure B-1: Percent error vs BMI using the difference method ...................................48

Figure B-2: Percent error vs BMI using the ratio method.......................................48

Figure B-3: Percent error vs change in anatomical LL using the difference method.......49

Figure B-4:1Percent error vs change in anatomical LL using the ratio method..............49

Figure B-5: Change in PT vs Change in LL at heal strike .........................................50

Figure B-6: Change in PT vs Change in LL while standing ......................................50 


\section{LIST OF ABBREVIATIONS}

$\begin{array}{ll}\text { ASIS } & \text { Anterior Superior Iliac Spine } \\ \text { CC } & \text { Correction Constant } \\ \text { CF } & \text { Correction Factor } \\ \text { DICOM } & \text { Digital Imaging and Communications in Medicine } \\ \text { PF } & \text { Pelvis Flexion } \\ \text { LBP } & \text { Lower Back Pain } \\ \text { LL } & \text { Lumbar Lordosis } \\ \text { LF } & \text { Lumbar flexion } \\ \text { MRI } & \text { Magnetic Resonance Imaging (MRI) } \\ \text { PSIS } & \text { Posterior Superior Iliac Spine } \\ \text { PT } & \text { Pelvic Tilt } \\ \text { SI } & \text { Sacroiliac } \\ \text { UTHSC } & \text { University of Tennessee Health Science Center }\end{array}$




\section{CHAPTER 1. INTRODUCTION}

\section{Significance of Research}

Lower back pain (LBP) has become one of the most prevalent health issues afflicting Americans. It has been reported that $84 \%$ of people will experience LBP at some point in their life, and $23 \%$ of the population will suffer from chronic lower back pain [1]. The costs associated with LBP are also substantial; data from a Medical Expenditure Panel Survey showed that Americans spent approximately 26.3 billion dollars on lower back pain treatments in 1998 [2]. Treatment costs only partially represents the financial burden of LBP, as LBP accounts for approximately $40 \%$ of work absences in the United States, second only to the common cold [3]. The cost associated with work absences due to LBP range from $\$ 50$ billion to $\$ 100$ billion annually [3].

While some cases of LBP can be a result anatomical abnormalities or neurological disorders, most cases have no known pathological cause and are referred to as nonspecific LBP [4]. In these treatment and its efficacy varies greatly. Some cases of LBP can be managed simply by increasing activity, avoiding certain movements, or with other conservative treatment modalities (massage, heat, ice). Stretching, specifically of the hamstrings, is also a common treatment approach [5, 6], because people with LBP have a higher incidence of hamstring tightness [7]. While several studies have been conducted on the link between hamstring flexibility and LBP, the relationship between LBP and hamstring tightness is still not completely understood. Radwan et al reported a positive correlation between hamstring tightness and severity of LBP [8]. One explanation is that hamstring tightness causes the pelvis to tilt posteriorly due to the hamstring's origin on the ischial tuberosity. A posterior pelvic tilt would cause the spine to flatten or become hypolordotic [9, 10], increasing risk for LBP [11], which is likely due to higher loads placed on the anterior structures of the spine including the intervertebral disc $[12,13]$.

Several studies have examined the effect of hamstring tightness on pelvic tilt and lumbar lordosis. Lopez-Minarro et al found that hamstring stretching will increase anterior pelvic tilt and lumbar flexion during sit and reach, but found no significant differences in the standing position [14]. Li et al found an increase in hip motion during forward bend following a hamstring stretching protocol, however that did not correspond to a change in lumbar motion [9]. Neither of these studies looked at the effect of hamstring tightness during gait or any other activities and only the latter used subjects with clinically tight hamstrings.

\section{Thesis Aim and Outline}

The goal of this study was to determine how lengthening hamstrings in subjects with clinically tight hamstrings changes the sagittal lumbar lordosis and sagittal pelvic tilt during gait, standing, sit-to-stand, and picking up an object off the floor. We 
hypothesized that hamstring lengthening would cause an increase in anterior pelvic tilt which would correspond to an increase in lumbar lordosis (LL). To allow accurate comparison of lumbar lordosis measurements from different sessions a correction method was developed and validated which accounts for marker placement error and soft tissue artifact.

This thesis is organized into four chapters. The remainder of chapter 1 will provide an overview of relevant anatomy, experimental equipment, and experimental measurements. Chapter 2 explains the lumbar lordosis correction method and results of the validation. Chapter 3 will discuss the change in spino-pelvic kinematics during gait, forward bend, straight legged ball pick up, and sit to stand after a hamstring stretching intervention. Finally, chapter 4 will give a general discussion and conclusion of all the results.

\section{Overview of Anatomy}

\section{Spine}

The spine consists of 33 individual vertebrae divided into five regions: cervical, thoracic, lumbar, sacrum, and coccyx (Figure 1-1) [15]. The lumbar region is made up of five independent vertebrae which create a concave curve posteriorly (Lordosis). The vertebral bodies of the lumbar spine are larger than the cervical and thoracic region allowing them to sustain the larger loads seen in the lumbar spine $[15,16]$. The intervertebral discs in this region are also thicker which allows more mobility. The facets allow movements of flexion, extension, and side bending, but limit rotation [15]. The fifth lumbar vertebrae attaches to the sacrum via the lumbosacral joint. The sacrum is composed of five, fused vertebrae. The sacrum is wedged shape and makes up the posterior portion of the pelvis. It connects to the hip bones via the sacroiliac joint which serve to transfer the load from the spine onto the pelvis, and ultimately on to the lower limbs [15].

Each vertebra consists of two main parts: the body (anterior) and vertebral arch (posterior) (Figure 1-2). The body is roughly cylindrical and supports the majority of the loads placed on the lumbar spine. The vertebral arch is connected to the body by two pedicles. The pedicles create a thickened junction with the lamina, which unite posteriorly to form the spinous process. The each junction of the lamina and pedicle has a superior and inferior articular facet which articulate against the facets of the superior and inferior vertebra [15]. These facet joints bear some load, their primary function is to guide the motion of the spine [16]. The opening between the body and vertebral arch is thevertebral foramen. Collectively, the vertebral foramina form the vertebral canal which serves to house and protect the spinal cord [15]. 


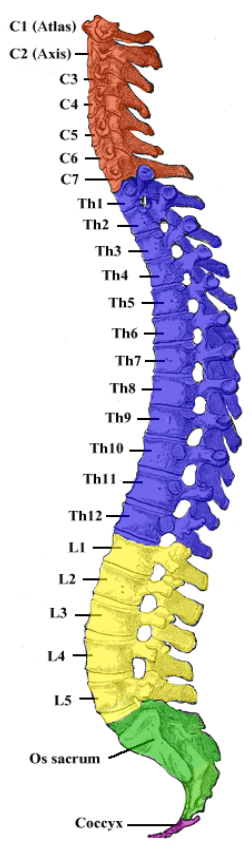

\section{Figure 1-1: $\quad$ Regions of the spine}

Vertebrae within the five regions of the spine (Cervical, thoracic, lumbar, sacral, and coccyx)

Source: Reprinted with kind permission from Wikipedia, The Free Encyclopedia:

https://en.wikipedia.org/wiki/Vertebral_column\#/media/File:Gray 111_-

Vertebral_column-coloured.png Accessed 5/31/16

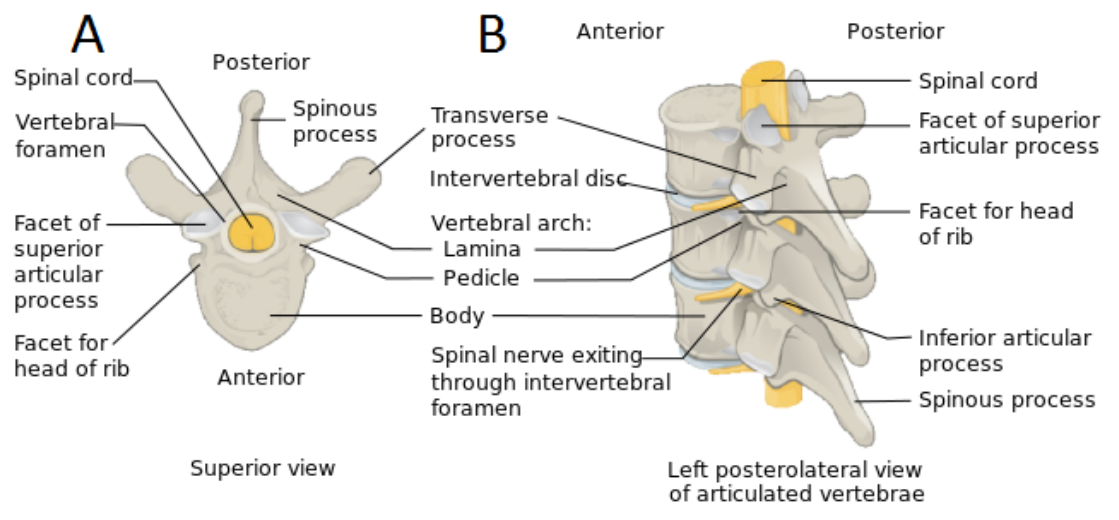

Figure 1-2: Anatomy of vertebrae

A) Superior view of vertebral body and vertebral arch with the spinous and transverse processes. B) Left posterolateral view of vertebrae

Source: Reprinted with kind permission from Openstax CNX:

http://cnx.org/contents/FPtK1zmh@6.27:4CMef3D9@4/The-Vertebral-Column. Accessed 5/31/16 
The body is surrounded anteriorly and posteriorly by the intervertebral discs (Figure 1-3). The intervertebral disc is composed of an inner and outer structure designed to hydrostatically absorb energy and distribute the load throughout the disc [17]. The inner structure, the nucleus pulposus, is composed of a collagen and elastin network that is imbedded in a highly hydrated gelatinous substance. A high concentration of hydrophilic proteoglycans gives the tissue a high water concentration resulting in incompressible fluid like mechanical behavior. When loads are placed on the spine it creates a hydrostatic pressure within the nucleus pulposus, which is transmitted into a tensile force in the outer region of the disc, the annulus fibrosis [17]. These characteristics allow the intervertebral disc to dissipate the load while permitting movement between the vertebrae [15].

The intervertebral disc is very proficient at performing its function as long as the nucleus pulposus remains highly hydrated. Unfortunately aging and excessive loading of the disc cause cellular changes in the nucleus pulposus which ultimately lead to fluid loss [18]. The position of the spine also affects the loading on the vertebral disc. When the lumbar spine is extended or hyperlordotic, more of the load is placed on the facet joints. When the spine flattens or becomes hypolordotic, the load is shifted onto the vertebral body and intervertebral disc [16]. As spine flexion increases, the nucleus pulposus is squeezed posteriorly which creates greater tensile stress on the posterior portion of the annulus fibrosis. Disc herniation occurs when fluid in the nucleus escapes through cracks or tears in the annulus fibrosis [15]. The escaped fluid may compress nerves causing back and leg pain. Increased loading on the disc may predispose individuals to acute pathologies such as disc herniation or chronic pathologies such as disc degeneration [18].

\section{Pelvis}

The pelvis is a ring shaped structure made up of two coxal bones, the sacrum, and the coccyx. Each coxal bone is actually composed of three bones which are fused together: the ilium, ischium, and pubis. The three bones fuse in a Y shaped joint at the center of the hip socket. The iliac crest makes up the superior edge of the hip bone with the anterior superior and the posterior superior iliac spines on each end of the iliac spine. Below the acetabulum is a bony arch which makes the obturator foramen through which several nerves and blood vessels pass [15]. The two ilia articulate with the sacrum at the sacroiliac (SI) joints which are reinforced by a capsule and strong network of ligaments including the sacrospinous and sacrotuberous ligaments which connect the sacrum to the ischial spine and ischial tuberosity respectively. The posterior sacroiliac ligaments connect the ilium the lateral sacral crest. While the SI joints main purpose is to stabilize the loads carried by the sacrum, it does allow some movement between the sacrum and hip bone. Adduction of the pelvis causes the sacral base to tilt anteroinferiorly and the sacral apex to tilt posterosuperiorly, while the iliac crests are pulled medially and the ischial tuberosities move laterally. This causes the diameter of the pelvic outlet to increase while the pelvic inlet decreases. Abduction causes the opposite movements, which result in the pelvic outlet to become smaller and the inlet to become larger. [15]. 


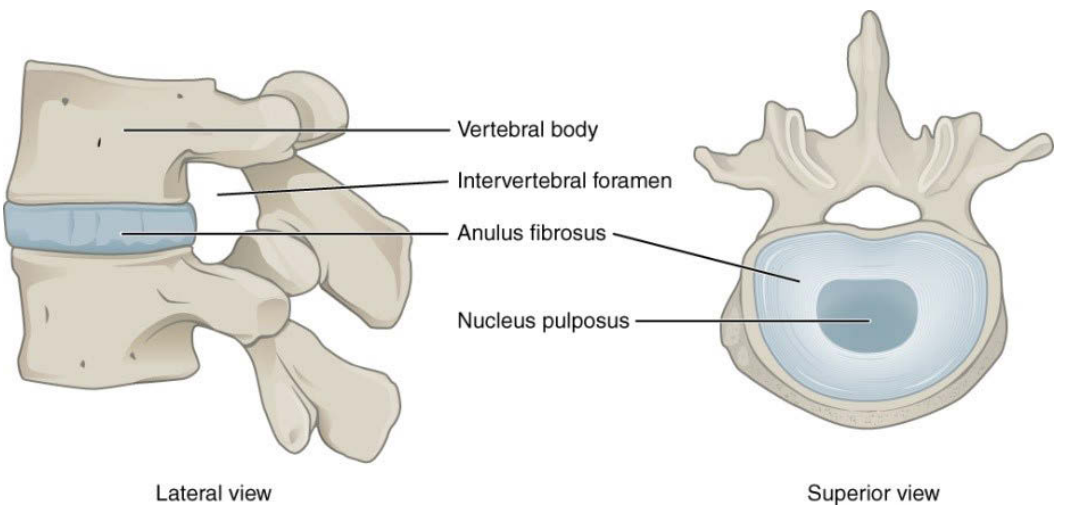

Figure 1-3: Anatomy of the intervertebral disc

Lateral and superior view of the intervertebral disc and vertebra

Source: Reprinted with kind permission from Openstax CNX:

http://cnx.org/contents/FPtK1zmh@6.27:4CMef3D9@4/The-Vertebral-Column.

Accessed 5/31/16 


\section{Hamstrings}

The hamstrings are a group of three muscles: the semitendinosus, semimembranosus, and biceps femoris; located on the posterior portion of the thigh (Figure 1-4). They collectively originate from the ischial tuberosity and insert on the tibia or fibula [19]. The semitendinosus and semimembranosus are both located medially and insert on the superomedial tibial shaft and posteromedial aspect of the tibial condyle respectively. The biceps femoris is located laterally and inserts on the head of the fibula [15]. Hamstring contraction primarily causes knee flexion and hip extension, though can also assist in rotation. The biceps femoris which inserts laterally acts in lateral rotation, while the medial attached muscles assist in medial rotation of the knee. If the thigh and shank are fixed, hamstring contraction will cause posterior pelvic tilt [15].

Insufficient stretching and exercise combined with prolonged sitting common with sedentary lifestyles has been associated with hamstring tightness. Because of the hamstrings' origin on the ischial tuberosity, tightness in the hamstrings may cause the pelvis to tilt posteriorly (Figure 1-5) [15]. It has been reported that a posterior pelvic tilt causes the spine to flatten or become hypolordotic which increases pressure on the vertebral disc $[10,12,13]$.

\section{Overview of Experimental Equipment}

\section{Motion Capture Laboratory}

Motion capture technology utilizes opto-electronic cameras (Qualisys, Gothenberg, Sweden) with a ring of near-infrared light emitting diodes to track the three dimensional location of retroreflective markers within the laboratory. Markers are placed on anatomical landmarks of interest and the cameras record 3D position of the markers as a subject performs an activity. The motion files are exported to Visual 3D (C-Motion, Germantown, MD, USA) which is used to generate a 6 degree of freedom rigid-linked segment model based on a static trial of each subject. The model is then applied to all motion activities to give the location and motion of each segment during the activity. Force plates are used to measure ground reaction forces and moments which are used to estimate joint power and moments.

\section{Qualisys Motion Capture System}

The University of Tennessee Health Science Center (UTHSC) Motion Analysis Laboratory utilizes 10 opto-electronic cameras consisting of models 100, 300, and 310 . The cameras are positioned along the perimeter of the lab to provide complete coverage of the raised 25 foot long platform in the center. Each camera is equipped with a ring of near-infrared light emitting diodes which pulse a narrow band of infrared light. Each camera has the ability to record light in the infrared and visible spectrum, however for this study only the infrared spectrum was used capturing at a rate of $100 \mathrm{~Hz}$. 


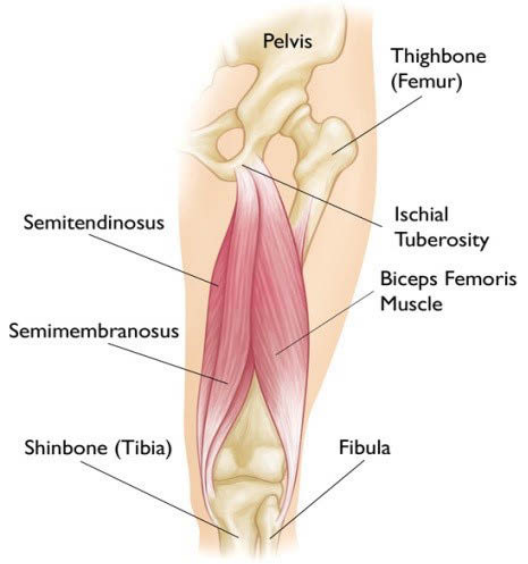

\section{Figure 1-4: Hamstring anatomy}

Origin and insertion of the hamstring muscles

Source: Reprinted with kind permission from AAOS,

http://orthoinfo.aaos.org/topic.cfm?topic $=$ a00408, accessed 5/24/16

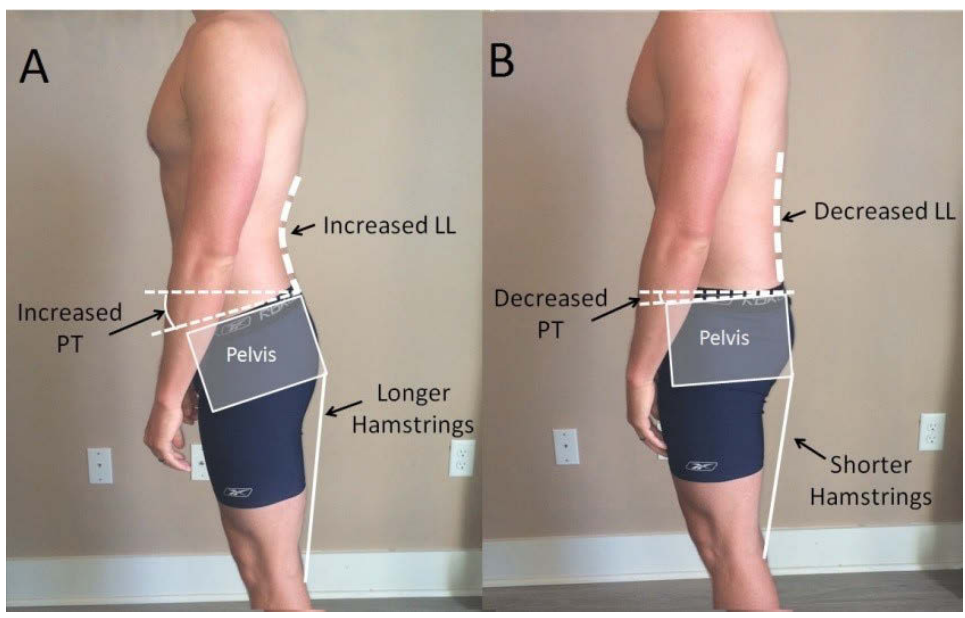

Figure 1-5: The effect of hamstring tightness on PT and $L L$

A) Subject with normal hamstring length B) Subject with tight hamstrings 
Qualisys Track Manager 10.0 (QTM) was used to record the three dimensional position of $12.7 \mathrm{~mm}$ and $9.5 \mathrm{~mm}$ retro-reflective markers placed on anatomical landmarks of the torso and lower extremities (Figure 1-6). The larger markers were used for the majority of landmarks, while the smaller markers served as filler markers on the spine. The cameras were calibrated prior to each testing by using a manufacturer's standard protocol to track marker position within a standard deviation of $0.5 \mathrm{~mm}$.

\section{Kinematics}

Markers were labeled in the motion files collected from QTM and then exported to Visual 3D for processing. Marker position was interpolated over a maximum of 10 frames using a $3^{\text {rd }}$ order polynomial, and low pass filtered at $7 \mathrm{~Hz}$ using a digital $4^{\text {th }}$ order Butterworth filter. A static trial of each participant standing with arms at $45^{\circ}$ was used to generate a rigid-linked segment model made up of eight geometric shapes (Figure 1-7). The foot, shank, and thigh of each leg were modeled as cones; and the pelvis and torso were be modeled by right elliptical cylinders. The locations of ankle and knee joint centers are given by the midpoints of the malleoli and epicondyles, respectively.

The Visual 3D model was used to define the landmarks of center anterior superior iliac spine (CASIS) and center posterior superior iliac spine (CPSIS). The angle created by the line connecting CASIS and CPSIS and vertical was defined as anterior pelvic tilt (PT) (Figure 1-8). The acute angle formed by the orthogonal lines extending from the vertebral endplates of interest is defined as the cobb angle, which is the gold standard for measuring lumbar lordosis (LL). When utilizing motion capture the vertebral endplates are not visible, so it is necessary to use skin markers placed on the spinous processes of interest to track spinal motion. Previous studies have validated the skin marker measurements with radiograph measurements and found that the skin marker motion correlates strongly with the anatomical motion measured on the radiograph. However, due to marker placement error and soft tissue artifact, it is difficult to compare absolute values of skin marker measurements between subjects $[20,21]$. For this study LL was defined as the acute angle between the lines connecting the markers on T12 to L2 and S2 to L4. To allow comparison between testing session, a correction method was used to correct for marker placement error and soft tissue artifact. This method will be discussed in detail in Chapter 2.

\section{AMTI Force Plates}

The motion analysis lab also utilizes three AMTI force plates (AMTI, Watertown, MA, USA) which are flush with the walking platform. The force plates measure ground reaction force which can be used to estimate external joint moments and powers, however for this study were only used to define events within the activity. The force plates use Wheatstone bridge principles and strain gauges to measure forces and moments in three dimensions at a sample rate of $100 \mathrm{~Hz}$. 


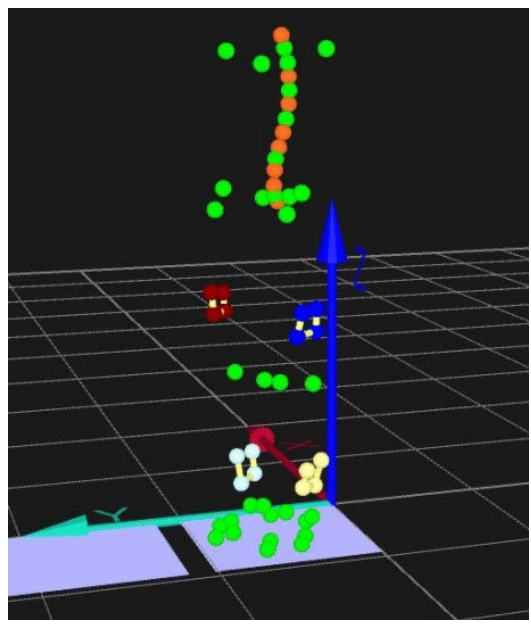

Figure 1-6: QTM marker set and Visual 3D model

Markers placed on anatomical landmarks and recorded by QTM

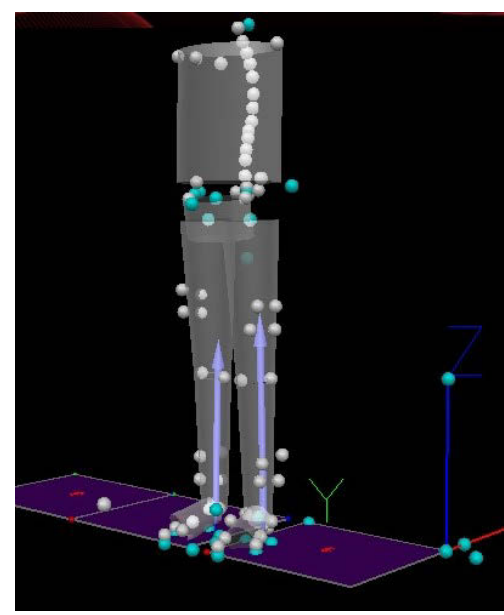

Figure 1-7: Eight segment model applied to static trial in Visual 3D 


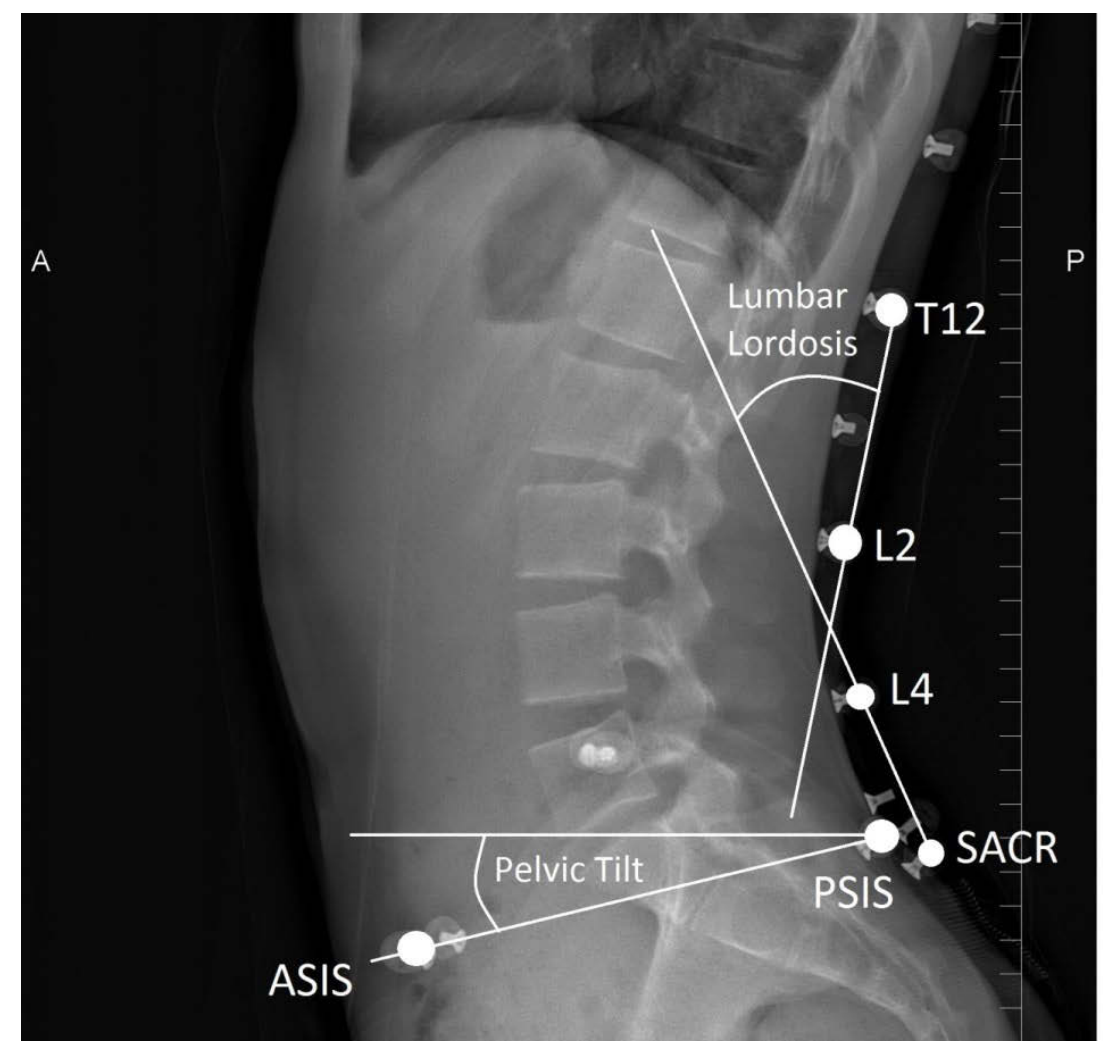

Figure 1-8: Pelvic tilt and lumbar lordosis angle measurements

Pelvic tilt derived from the markers on anterior superior iliac spine and posterior superior iliac spine. Lumbar lordosis derived from the markers on T12, L2, L4, and sacrum. 


\section{EOS Bi-Planar X-Ray System}

The EOS Bi-planar X-Ray System (EOS Imaging, Cambridge, MA, USA) captures simultaneous, 1:1 scale, sagittal and frontal X-ray images of the subject in standing position. EOS accomplishes this by using two perpendicular X-ray beams collimated in two thin, horizontal, fan shaped beams and two detectors. The X-rays and detectors are mounted on a $\mathrm{C}$-arm which moves vertically through the range of the scan, which is capable of taking a full body image. Another feature of the EOS system is the low exposure of radiation while producing and image quality equal to standard radiography.

A trained technician uses SterEOS 3D modeling software (EOS Imaging, Cambridge, MA, USA) to reconstruct a virtual 3D spine from the two dimensional scans. The reconstruction is created by matching the three dimensional shape and location of the bones of a generic, normal skeleton to the two dimensional scans by identifying 3D coordinates of specific landmarks on each bone. EOS 3D reconstruction has been validated for various bones including the vertebrae which resulted in an average discrepancy of $0.9 \mathrm{~mm}[22]$.

Spine model generates several measurements including lumbar lordosis, thoracic kyphosis, vertebral orientations, and pelvic parameters (Figure 1-9). The measurements of interest for this study are the L1/L5 lumbar lordosis and L1/S1 lumbar lordosis. The standard measurement of lumbar lordosis is the Cobb angle which is defined as the acute angle formed by the orthogonal lines extending from the vertebral endplates of interest. L1/L5 lumbar lordosis is derived from the Cobb angle created from the superior endplate of L1 and inferior endplate of L5. L1/S1 lumbar lordosis is derived from the Cobb angle created by the superior endplate of L1 and the superior sacral endplate. The EOS measurements were used to develop a relationship between marker based LL and anatomical based LL, which was used to correct the LL measurements from the motion capture lab. This will be explained in detail in Chapter 2. 


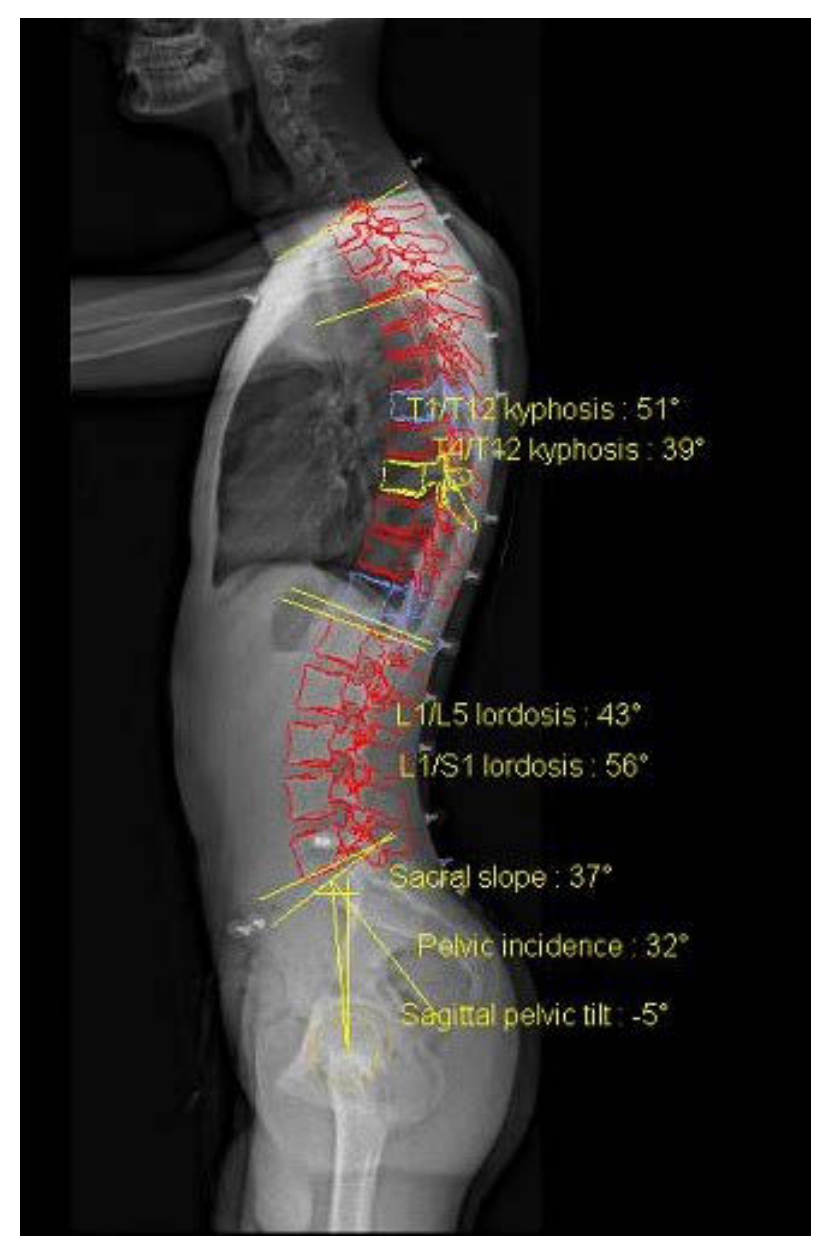

Figure 1-9: EOS spinal and pelvic measurements

Sagittal spinal and pelvic measurements generated from SterEOS reconstruction 


\section{CHAPTER 2. A NOVEL METHOD FOR CORRECTING MOTION LAB LUMBAR LORDOSIS MEASUREMENTS UTILIZING AN EOS BI-PLANAR X- RAY SYSTEM}

\section{Introduction}

Many studies have concluded that the movement of skin markers accurately describes the movement of the underlying vertebrae [20, 21, 23]. Morl and Blickhan utilized magnetic resonance imaging (MRI) of nine subjects at five different positions of lumbar flexion and found a strong relationship between the translation of the external skin markers and the translation of corresponding vertebrae [21]. Hashemirad et al. performed a similar study utilizing radiograph techniques and found that skin markers can confidently be used to estimate the motion of the lumbar spine [20]. Both researchers report that the use of skin markers is not adequate for measurement of absolute LL, mainly due to marker placement error and soft tissue artifact.

Tissue interface and distribution vary greatly between subjects making it impossible to infer absolute values of LL without internal imaging. There is also a large amount of error introduced from marker placement as the markers are placed by palpation. A marker validation study which utilized the same marker set and methods as the current study found that only $36 \%$ of spine marker placements accurately represented the position of the underlying vertebrae. The study noted that $81 \%$ were within one vertebra, and the remaining 19\% were within two vertebrae [23]. Schmid et al performed a similar study and reported that the mean distance of inaccurate marker placement was $19.3 \pm 18.6 \mathrm{~mm}$ above or below the targeted level [24]. Due to the curvature of the spine, misplacement of markers will significantly alter marker based LL measurements taken in a motion capture lab. In a subject with a LL measurement of $35^{\circ}$ misplacing a marker by $2 \mathrm{~cm}$ could change the LL measurement by $10^{\circ}$ resulting in an error of approximately $30 \%$. This error will be greater on subjects with greater LL.

Whittel and Levine found that the use of skin mounted rigs gave more repeatable and accurate measurement of LL than using additional markers mounted directly on the surface of the skin [25]. However, this study did not touch on the accuracy of marker placement or the repeatability of correct marker placement for follow up testing sessions. The error in marker placement makes it impossible to compare LL measurements of within subjects during different testing sessions. Because permanently marking marker locations on a subject is not an option, another method had to be developed to allow comparison of pre and post intervention LL measurements.

The use of the EOS Bi-Planar X-Ray system allows the comparison of marker based LL to anatomical based LL which is measured on the radiograph or calculated from the 3D SterEOS model. Assaf found that when a change was implemented in the spinal curvature, the change in marker based measurement accurately described the change in vertebral measurement [23]. Since the change between the two measurements remains constant, the difference between the two measurements on the radiograph will give a 
correction constant (CC) which can be added to the marker based LL measured in the lab to give a corrected LL. Alternatively, if the relationship does not stay constant it may be better represented by a ratio of the two curves which would give a correction factor (CF) that can be used to correct laboratory LL measurements.

The purpose of this portion of the study was to determine whether the ratio correction method or the difference correction method more accurately corrects marker based lumbar lordosis measurements. This was determined by using radiographs of subjects in two different spinal positions, and using the $\mathrm{CF}$ and $\mathrm{CC}$ calculated from the first scan to calculate the corrected LL from markers on the second scan. The corrected LL was then compared to the anatomical based LL.

\section{Methods}

\section{Participants}

After obtaining IRB approval (Appendix A), twenty-one healthy subjects (12 male, 9 female) with no history of LBP, spine injuries or defects were recruited for the study. Participants ranged between 21-35 years of age with a mean \pm SD of 23.6 years \pm 3.3 years. Average weight and height were $71.1 \mathrm{~kg} \pm 13.8 \mathrm{~kg}$ and $1.73 \mathrm{~m} \pm .067 \mathrm{~m}$ respectively, resulting in an average BMI of $23.5 \pm 3.3$.

After obtaining IRB approved informed consent, subjects were given a physical therapist evaluation by a licensed physical therapist to screen for any undiagnosed physical limitations. To ensure no pregnant women would be exposed to extra radiation caused by the EOS bi-planer X-ray scan, all females were required to take a urine pregnancy test. None of the pregnancy tests indicated pregnancy, and therefore no subjects were removed due to this criterion

\section{Data Collection}

Following the evaluation, a physical therapist placed 58 reflective, radio-opaque markers by palpation on anatomical landmarks of the torso and lower extremities. The spine was defined by markers on the C7, T4, T7, T10, T12, L2, L4, and S2. Filler markers were placed in between spine markers when possible to better define the spinal curvature. The pelvis was defined by markers placed on the anterior superior iliac spine (ASIS), posterior superior iliac spine (PSIS), and iliac crest. The leg was defined by markers placed on the thigh, lateral and medial femoral epicondyles, shank, and lateral and medial malleolus. The feet were defined by markers on the calcaneus, dorsum, $1^{\text {st }}$ metatarsal, $5^{\text {th }}$ metatarsal, and big toe (Figure 2-1). The subject then performed several activities with and without a custom made hyper-tensioned clavicle strap designed to alter LL. These activities were recorded in the motion capture laboratory for a larger study. 


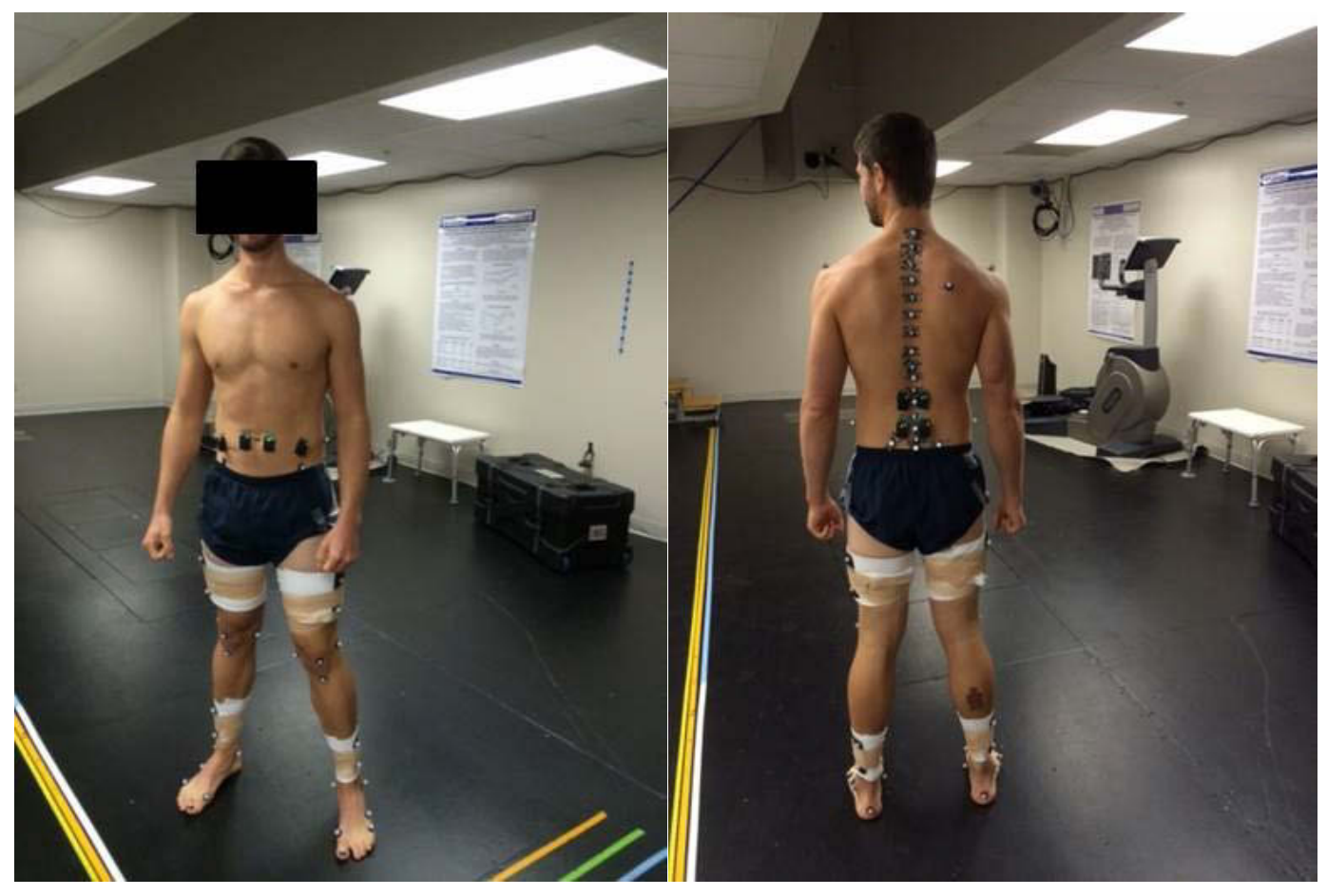

Figure 2-1: Subject with reflective markers on torso and lower extremities 
The clavicle strap was developed with the assistance of Spears Prosthetics \& Orthotics/Rehab Services and designed to alter spinal curvature without affecting the pelvis or interfering with markers on the spine. The clavicle strap was tensioned to the limit of the subject's comfort level, and the position was marked with a pen. Following the trials, the markers on the lower extremities and clavicle strap were removed and the subject was taken to Le Bonheur Children's Hospital to have two bi-planar X-ray scans performed.

At Le Bonheur the subject was positioned in the EOS scanner in a standing position with their hands lightly grasping a radio-translucent handle which was positioned so the subject's humeri would not interfere with the sagittal view of the spine. The subject was scanned from $\mathrm{C} 1$ vertebra to mid-thigh. Following the first scan, the orthotic was placed on the subject and tensioned to the same setting marked for the motion capture trials. The subject then received a second scan in the same position as the first scan.

\section{Data Analysis}

Three different anatomical based LL measurements were investigated. Two of them were generated from EOS reconstruction and the third measurement was the clinical LL measurements taken from the radiograph. For the EOS measurements, the EOS scans were used to generate a $3 \mathrm{D}$ reconstruction of the spine and pelvis by the one of two trained Le Bonheur technicians using SterEOS software. A report is then generated from the reconstruction which contains, among other measurements, the L1/L5 and L1/S1 lumbar lordosis.

For the clinical measurement, the scans from EOS were exported in Digital Imaging and Communications in Medicine (DICOM) format. An orthopedic resident used Surgimap (Nemaris Inc, NY, USA) to make manual measurements of L1/L5 lumbar lordosis from the sagittal images as described in detail in Chapter 1 (Figure 2-2).

The spinal markers were also clearly displayed on the sagittal and frontal views. The markers are constructed with a radio-opaque centrally located screw. Using the screw as the centroid of the marker, marker based LL lordosis could be measured on the scans in the same method as it is measured in the motion capture laboratory. The resident measured the acute angle formed by the lines connecting the screw tips of T12 to L2 and S2 to L4.

Two different relationships between the anatomical based measurements and the marker based measurements were investigated. The difference method uses a correction constant (CC) which is simply the difference between the anatomical based LL and the marker based LL. This method assumes that the difference between the two measurements will remain constant through varying degrees of LL as described by Assaf [23]. The difference between the two will give the $\mathrm{CC}$ which would be added to all marker based LL measurements to calculate a corrected LL. 


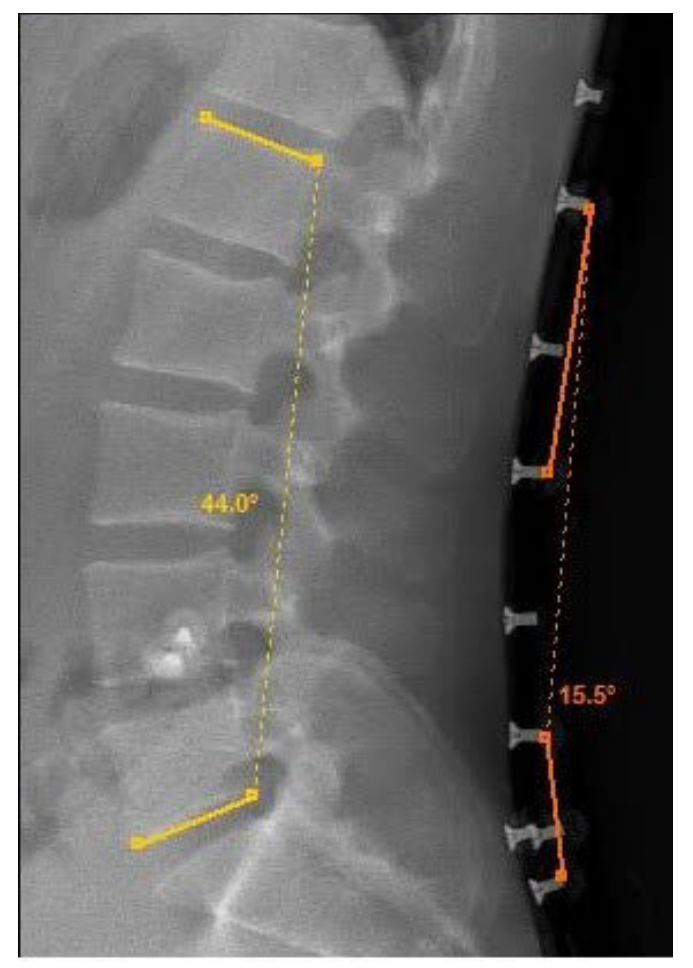

Figure 2-2: Clinical LL and marker based LL on radiograph

Clinical L1/L5 LL (Yellow) and marker based LL (Orange) taken from the radiograph 


$$
\begin{aligned}
& C C=L L_{\text {Anatomical }}-L L_{\text {Marker }} \\
& L L_{\text {Corrected }}=L L_{\text {Marker }}+C C
\end{aligned}
$$

The ratio method takes the anatomical based measurement divided by the marker based measurement to give a correction factor (CF). The correction factor is then multiplied by all marker based LL measurements to give a corrected LL. This method assumes that the difference between the two measurements does not remain constant through changes in spinal curvature; however the ratio of the two measurements will remain constant.

$$
\begin{gathered}
C F=\frac{L L_{\text {Anatomical }}}{L L_{\text {Marker }}} \\
L L_{\text {Corrected }}=L L_{\text {Marker }} * C F
\end{gathered}
$$

To validate and compare the two methods, the CF and $\mathrm{CC}$ was calculated from the scan without the clavicle strap, and then applied to the marker based LL from the second scan with the clavicle strap to find a corrected LL. The error and percent error between the corrected LL and the anatomical LL were then calculated. To determine which anatomical based measurement best correlates to the marker based measurements, the validation was performed on all anatomical measurements: EOS derived L1/L5 LL, EOS derived L1/S1 LL, and clinical LL measurements from the radiograph.

$$
\begin{gathered}
\text { Error }=A b s\left(L L_{\text {Anatomical }}-L L_{\text {Corrected }}\right) \\
\text { Percent Error }=\frac{A b s\left(L L_{\text {Anatomical }}-L L_{\text {Corrected }}\right)}{L L_{\text {Anatomical }}} * 100
\end{gathered}
$$

A paired t-test was used to determine the difference in the percent error of the ratio and difference methods using all three measurements.

\section{Results}

The clavicle strap resulted in an average absolute change of $4.6^{\circ} \pm 4.0^{\circ}$ (Mean \pm $\mathrm{SD})$ as measured from the EOS reconstruction. Two subjects were discarded from the validation study because markers were not visible in one or both of the scans. EOS reconstructions were not made for three individuals due to spinal abnormalities; therefore the validation for the EOS based measurements utilized the measurements from the remaining 16 participants. The difference method gave lower error values than the ratio method however the difference was only significant when using the L1/L5 measurements $(\mathrm{p}=0.004)$. The difference method using the L1/L5 measurements gave the lowest average percent error of $6.2 \% \pm 4.8 \%$. The lowest error using the ratio method was using the L1/S1 measurement resulting in an average percent error of $8.9 \% \pm 6.1 \%$. The 
difference in error between the two methods was only significant when using the L1/L5 measurements $(p=0.004)$.

The clinical measurements of nineteen subjects were used for the clinical measurement validation. These subjects had an average, absolute LL change of $4.2^{\circ} \pm$ $4.0^{\circ}$ from the clavicle strap. The difference method again resulted in lower average error $(7.5 \% \pm 5.3 \%)$ however the difference between the two methods was not significant $(\mathrm{p}=0.17)$ The ratio method gave an average percent error of $9.4 \% \pm 5.8 \%$ (Table $2-1)$. The error and percentage error of each method was also plotted with subject BMI and absolute change in LL to determine if either of these factors significantly contributed to the error, however no correlation was found (Appendix B).

\section{Discussion}

The most accurate method for lumbar lordosis correction was the difference method utilizing the EOS L1/L5 LL measurement. The difference method consistently gave better results than the ratio method regardless of the measurement technique however this was only significant using the L1/L5 measurements. This result is not surprising, given that other studies have reported a change in marker based LL that was consistent with the change in anatomical based LL. Assaf et al. reported a mean difference of $3.2^{\circ} \pm 2.2^{\circ}$ which is very close to the amount of error found in this correction method [23]. While it would be desirable to have an error lower than $6.2 \%$, this error is lower than what would be introduced by error in marker placement which could easily result in an error of greater than $30 \%$ based on the example given earlier. Even the largest error reported was $18 \%$ which is less than the error that could result from incorrect marker placement.

The EOS measurements also generally gave better results than the clinical measurements, although the differences in error were minimal. There are three participants that were included in the clinical measurement validation which were not included in the EOS measurement validation because they did not have reconstructions available, however these did not account for the difference. The average error for those three subjects was $7.5 \%$ which is exactly what the overall error was for the clinical measurements. The difference is likely due to the human error introduced by making the clinical measurements on the radiograph. There is some subjectivity when drawing the orthogonal lines from the endplates of the vertebra and measurements can vary several degrees between measurements by the same person. The EOS reconstruction involves lining up several landmarks for each segment, which reduces the amount of human error introduced. Error could also be introduced to the clinical measurements if the subject is slightly rotated in the EOS scanner. The SterEOS model accounts for this rotation in its measurements, however it would alter angles measured from the sagittal radiograph. This is a potential source of error for all methods, as the marker based measurements are all taken from the sagittal scan. 
Table 2-1: $\quad$ Error in LL correction method (Mean \pm SD)

\begin{tabular}{lccccc}
\hline & \multicolumn{2}{c}{ Ratio Method } & & \multicolumn{2}{c}{ Difference Method } \\
\cline { 2 - 3 } \cline { 5 - 6 } LL Measurement & Error & Percent Error & & Error & Percent Error \\
\hline EOS L1/S1 LL & $5.1^{\circ} \pm 4.0^{\circ}$ & $8.9 \% \pm 6.1 \%$ & & $3.5^{\circ} \pm 2.6^{\circ}$ & $6.6 \% \pm 4.9 \%$ \\
EOS L1/L5 LL & $5.2^{\circ} \pm 3.6^{\circ}$ & $10.4 \% \pm 6.5 \%$ & & $3.2^{\circ} \pm 2.8^{\circ}$ & $6.2 \% \pm 4.8 \%$ \\
Clinical LL & $4.1^{\circ} \pm 2.4^{\circ}$ & $9.4 \% \pm 5.8 \%$ & & $3.2^{\circ} \pm 2.1^{\circ}$ & $7.5 \% \pm 5.3 \%$ \\
\hline
\end{tabular}


Despite the limitations of the sagittal X-ray, it is important to consider it would be a much cheaper and more accessible alternative to the EOS Bi-Planar scan. Given that there was only an error difference of $0.9 \%$ between the clinical and the EOS L1/L5 measurement, the clinical measurement on a sagittal X-ray would be a valid alternative to an EOS scan. It was thought that higher BMI would result in a larger error in the corrected LL due to the increase in soft tissue between the skin and the spinous processes. However this so it is likely that the skin marker movement would not correlate as well with the spinal movements. However, this study did not include any extremely high BMI subjects with the highest being 29.9; and the average being 23.5. This method may lose accuracy for obese subjects which also tend to have a greater amount of marker placement error because spinous process palpation becomes more difficult [26].

It was also surprising that subjects that experienced greater changes in LL did not result in larger errors from the correction factor. A limitation to this validation is that the amount of change in LL for all individuals was relatively small. The average change was only $4.6^{\circ}$ with the largest change being $15^{\circ}$. This validation utilized scans that were

already taken for another study, so it was not possible to take scans with greater ranges of flexion. It would have been desirable to validate this method on subjects at several greater degrees of flexion; however this would require several more scans and reconstructions resulting in greater radiation exposure for each subject. Further studies should include validation of this method at greater degrees of lumbar flexion and extension. It is likely that this method would lose its validity at greater degrees of flexion, since the relationship is based on a standing position, but for standing activities such as walking, it remains an option for comparing absolute values of LL.

\section{Conclusion}

The lumbar lordosis correction method using the difference method allows more accurate measurement of absolute LL than just using the raw marker based LL measurements taken from a motion capture lab. Given that error in marker placement can give errors greater than $30 \%$, this method allows for more accurate comparison of absolute LL and measurements taken from multiple testing sessions. While the EOS measurements gave slightly lower error, the difference was minimal, and taking one sagittal X-ray is a much more economical option for most labs. However, it is important to keep in mind the limitations of this method. Although $6.2 \%$ error is likely better than using raw LL values from a gait lab, it is still not adequate for detecting small differences in LL measurements. Also, because it was only verified using subjects with healthy BMI's with a limited range of spinal flexion, this method should only be used on individuals with a BMI below 30 and for activities where LL is within $10^{\circ}$ of the LL measured in the scan. For the remainder of the thesis, the use of the correction method will be limited to activities where the laboratory based LL is within $10^{\circ}$ of the LL measured on the radiograph. 


\section{CHAPTER 3. EFFECT OF HAMSTRING LENGTHENING ON PELVIC TILT AND LUMBAR LORDOSIS}

\section{Introduction}

The hamstrings are grouped into medial (semimembranosus and semitendinosus) and lateral (biceps femoris) muscles. They function to create knee flexion, hip extension, and posterior tilt of the pelvis [15] All three muscles originate on the ischial tuberosity of the pelvis and insert on the bones on the tibia or fibula [15]. Because of their origin on the pelvis, short hamstrings can cause the pelvis to tilt posteriorly. A posterior pelvic tilt can also cause the lumbar spine to flatten, becoming hypolordotic [10]. As explained in Chapter 1, a hypolordotic lumbar spine may result in increased load on the intervertebral disc, which would increase the risk of LBP $[11,13]$.

While the relationship between hamstring length, pelvic tilt, and lumbar lordosis has been widely reported, it is still a subject of current debate. It has been reported that tighter hamstrings cause a decrease in range of anterior pelvic tilt and increase lumbar flexion during activities that involve trunk flexion with knees straight, however the findings are less conclusive in an upright or walking position [9, 14, 27]. Gajdosik et al compared thirty male participants divided into three groups based on hamstring length. They reported decreased anterior pelvic tilt and increased lumbar flexion during forward bend in the group with short hamstrings, however no difference was found in pelvic tilt or lumbar lordosis in the standing position [27]. Lumbar lordosis and pelvic tilt at standing can vary greatly between healthy individuals; therefore it would be difficult to detect statistically significant differences between the groups. To determine the true effect of hamstrings on pelvic positioning, it may be more effective to perform an intervention to change the hamstring length.

Lopez-Minarrro et al. determined the acute effects of hamstring stretching on spinal curvature and pelvic tilt during standing and sit-and-reach before and after a hamstring stretching session. During sit-and-reach participants achieved greater pelvic tilt and lumbar flexion than they had achieved before stretching; however there was no difference in standing [14]. This study looked at changes after only one stretching session and it has been shown that a single session does not cause enough tissue deformation to produce a permanent, adaptive change [28]. Another limitation is that the participants didn't necessarily have tight hamstrings to begin with; therefore their hamstrings likely were not contributing to an altered pelvic or spinal posture during standing before the intervention.

Li et al. conducted a study using thirty-nine subjects with tight hamstrings which were randomly assigned to a stretching group or control group. The stretching group completed a stretching regimen which consisted of three weeks of daily stretching. Pre and post intervention PT and lumbar angle were compared for each subject during standing and forward bend activities. Similar to other studies, Li et al. reported an increase in anterior PT during forward bend, but not during standing. While there was an 
average increase in PT during standing, this increase was not statistically significant [9]. $\mathrm{Li}$ et al. also noted that not all subjects in the stretching group increased in hamstring length, which indicates that the stretching protocol may not have been followed or may not have been effective. Furthermore, the lumbar angle measurement utilized a marker which was placed on L1 by palpation; however several studies have shown the inconsistency of marker placement by palpation which would invalidate any of lumbar angle comparisons $[23,24]$.

The challenge with investigating standing positions is that participants will naturally have some variation in their posture between measurements which will make it difficult to measure true changes. Looking at an activity of daily living such as walking may give better repeatability between measurements. Gait is not only the most common activity humans perform; it also recruits and stretches the hamstring muscles. While standing the hip is in a neutral position, therefore hamstring tightness may not have any effect. During heel strike, the knee is extended and the hip is flexed, therefore the hamstring is in a more stretched state. Whitehead et al. attempted to simulate the effect of hamstring tightness during gait by using a brace which consisted of a pelvic band and a tibial band which were connected posteriorly by a nylon strap. The strap could be adjusted to simulate multiple degrees of hamstring tightness. Once popliteal angle exceeded $85^{\circ}$, the participants experienced decreased anterior pelvic tilt, speed, and stride length [29]. To the authors knowledge, there are no studies published which examine the effect of hamstring stretching on pelvic tilt and lumbar lordosis during gait in individuals with tight hamstrings.

Therefore, the purpose of this study was to determine the effect of hamstring stretching on LL and PT in a cohort of subjects with clinically tight hamstrings during several activities including walking, forward bend, ball pick up, and sit to stand. Our hypothesis was that hamstring stretching would cause increased anterior PT and decreased LL. This was tested by having participants with tight hamstrings perform the activities of interest in a motion capture laboratory. After completion of a six week hamstring stretching protocol, the participants were brought back into the motion capture lab to repeat the activities.

\section{Methods}

\section{Subject Recruitment}

Subjects with reported tight hamstrings were recruited for the study through word of mouth and email announcements at the University of Tennessee Health Science Center (Memphis,TN). Subjects had to be between the ages of 18-45 years old and have no history of lower back injury or surgery. Before enrollment, subjects were screened by a physical therapist and assessed for adequate hamstring tightness. To eliminate the possibility of exposing any pregnant women to extra radiation from the EOS Biplanar XRay system, all women were required to take a urine pregnancy test to confirm they were 
not pregnant. No participants were excluded due to this criterion. Ten subjects (10 male, 2 female) were enrolled in the study and signed an IRB approved consent form. The demographics of subjects are summarized in Table 3-1.

\section{Physical Therapy Evaluation}

Following consent, subjects were given a brief physical therapy evaluation by a trained physical therapist. Height, weight, leg length, hip flexibility and hamstring flexibility were measured. Hamstring flexibility was determined my passive straight leg raise and passive knee extension with hip at $90^{\circ}$. Passive straight leg raise (SLR) was performed by placing the subject in supine and raising one leg to reported discomfort. The angle between the leg and horizontal was then measured. The passive knee extension was performed with the subject in supine and one limb passively moved to $90^{\circ}$ hip flexion. Each knee was independently, passively extended to reported discomfort and the angle between shank and vertical (popliteal angle) was measured (Figure 3-1). A popliteal angle (PA) greater than $25^{\circ}$ was required to participate in the study.

\section{Data Collection}

Following the evaluation, a physical therapist placed 58 reflective markers by palpation on anatomical landmarks of the torso and lower extremities. The spine was defined by markers on the C7, T4, T7, T10, T12, L2, L4, and S2. Filler markers were placed in between when possible to better define the spinal curvature. The pelvis was defined by markers placed on the anterior superior iliac spine (ASIS), posterior superior iliac spine (PSIS), and iliac crest. The leg was defined by markers placed on the thigh, lateral and medial femoral epicondyles, shank, and lateral and medial malleolus. The feet were defined by markers on the calcaneus, dorsum, $1^{\text {st }}$ metatarsal, $5^{\text {th }}$ metatarsal, and big toe. A static trial was taken in front of the opto-electronic cameras with the subject standing and arms positioned $45^{\circ}$ from the torso. The subject then performed walking, sit to stand from a bench, forward bending and extension, and picking up a weighted ball with straight legs.

\section{Walking}

Subjects were instructed to walk at a self-selected pace across the platform with three embedded force plates until at least five clean foot strikes were made with each foot on the force plates.

\section{Sit to Stand}

Subjects stood in front of an 18 " high bench with one foot on each force plate. Subjects were instructed to sit on the bench, pause, and stand again without using their arms to assist (Figure 3-2). This cycle was repeated for 30 seconds for two trials. 
Table 3-1: $\quad$ Summary of subject demographics (Mean \pm SD)

\begin{tabular}{lcccc}
\hline \multicolumn{1}{c}{ Subjects } & Height $(\mathbf{c m})$ & Mass $(\mathbf{k g})$ & BMI $\left(\mathbf{k g} / \mathbf{m}^{\mathbf{2}}\right)$ & Age $($ years $)$ \\
\hline Male $(\mathrm{N}=10)$ & $178 \pm 5$ & $74 \pm 13$ & $23 \pm 3$ & $25 \pm 3$ \\
Female $(\mathrm{N}=2)$ & $165 \pm 7$ & $55 \pm 8$ & $20 \pm 1$ & $24 \pm 1$ \\
Total $(\mathrm{N}=8)$ & $176 \pm 7$ & $71 \pm 14$ & $23 \pm 3$ & $25 \pm 3$ \\
\hline
\end{tabular}

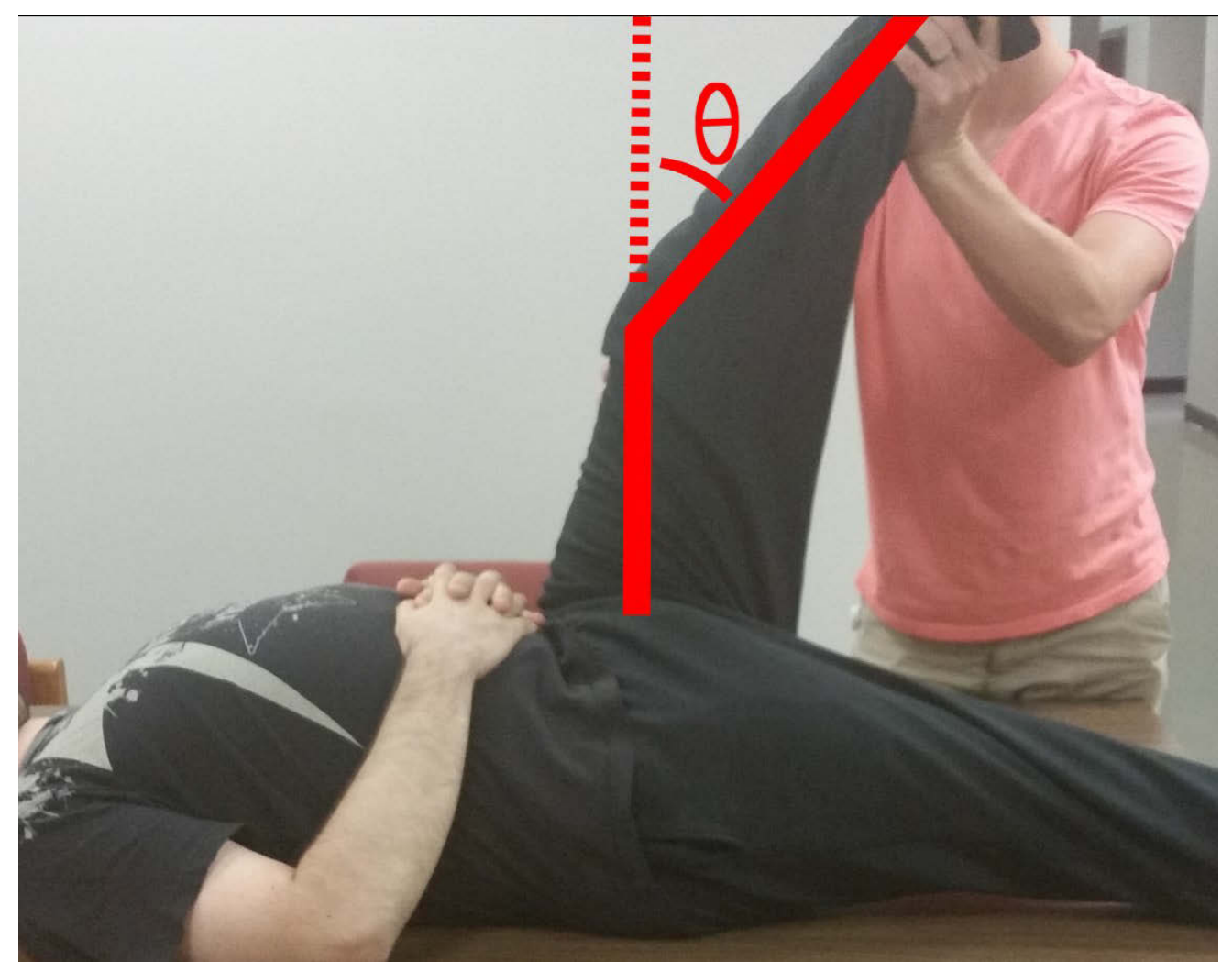

Figure 3-1: Measurement of popliteal angle

Measurement of PA during passive knee extension with hip at $90^{\circ}$ flexion 


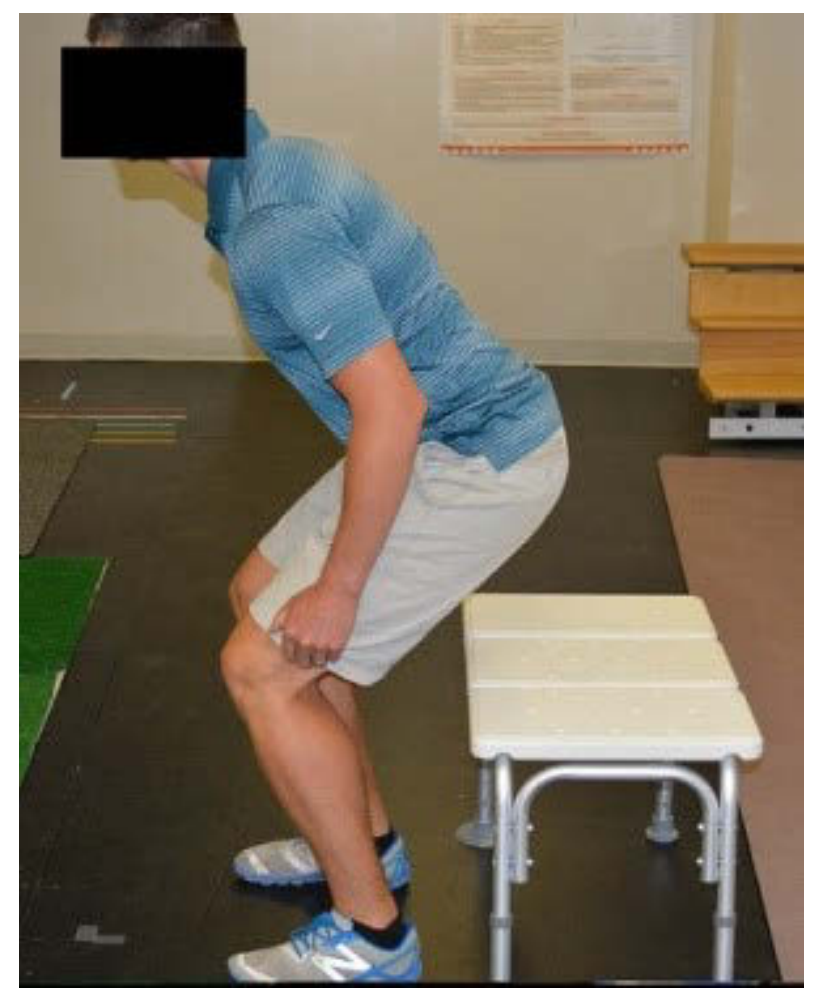

Figure 3-2: Subject performing sit to stand 


\section{Forward Bend}

Subjects stood normally with a foot on each foot plate and were instructed to bend forward and reach for the floor with knees in full extension. The subject then returned to standing and extended the torso and arms as far back as possible (Figure 3-3). This motion was repeated for 30 seconds for two trials.

\section{Ball Pick Up}

Subjects stood with a foot on each force plate. An $81 \mathrm{~b}$ ball was placed in front of their feet and the subjects bent at the waist and picked up the ball while trying to keep their knees as straight as possible. The ball was raised to waist level and then placed back on the floor while keeping their knees as straight as possible and returning to standing (Figure 3-4). This motion was repeated for 30 seconds for two trials.

\section{EOS Bi-Planar X-Ray}

After all the activities were completed the markers were removed from the lower extremities, and the subject was escorted to Le Bonheur Children's Hospital for a scan using the EOS Bi-planar X-Ray System. At Le Bonheur, the subject was positioned in the EOS scanner in a standing position with their hands lightly grasping a radiotranslucent handle. The handle was positioned so the subject's humeri would not interfere with the sagittal view of the spine. The subject was scanned from $\mathrm{C} 1$ to midthigh.

\section{Stretching Protocol}

Following testing, the subjects completed a six week hamstring stretching protocol to increase the length of their hamstrings. The first stretching session was completed with a physical therapist to ensure proper stretching technique. Subjects lied in supine position on the floor near a doorway or wall corner. The leg to be stretched was positioned on the wall while the opposite leg rested flat on the floor. The subject moved closer to the wall until a comfortable stretch was felt in each hamstring. When a stretching sensation was no longer felt, the subject slid closer to the wall. This position was held for 30 seconds, followed by a 10 second rest before repeating. The subjects performed this stretch four times on each leg resulting in a total of 2 minutes of stretching for each leg. The stretching protocol was performed twice a day, three days per week for 6 weeks. Each subject also attended group stretching session weekly which was supervised by a physical therapist to ensure proper form and measure progress. Following the stretching protocol the subject returned to the gait lab to repeat all base line tests reported earlier. 


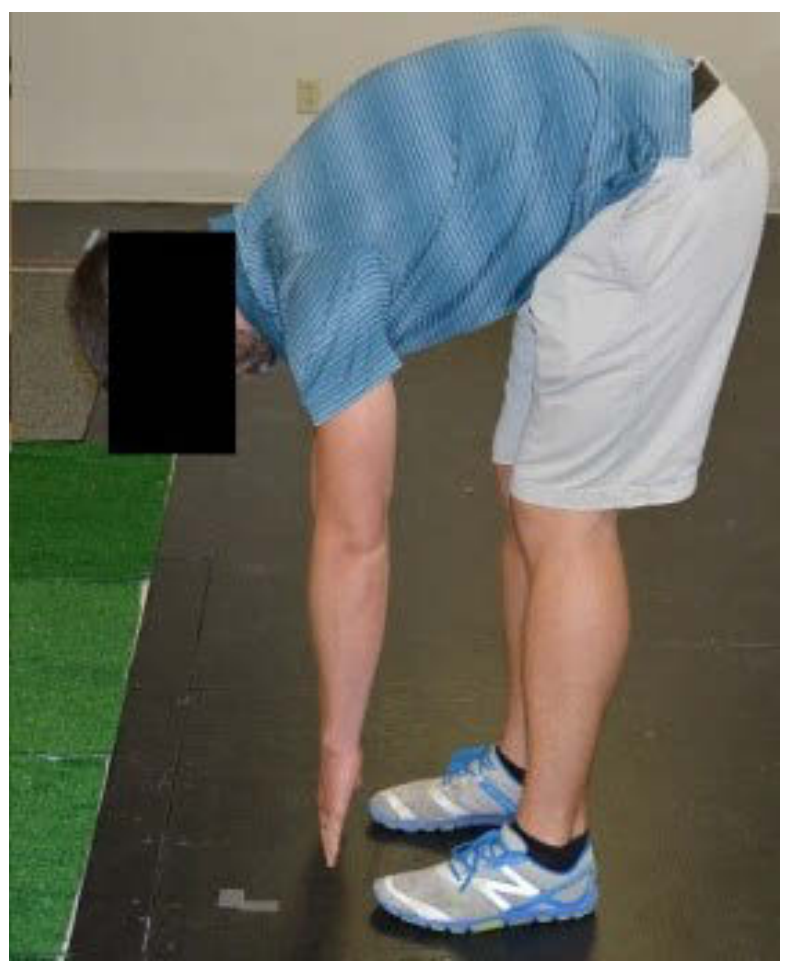

Figure 3-3: Subject performing forward bend

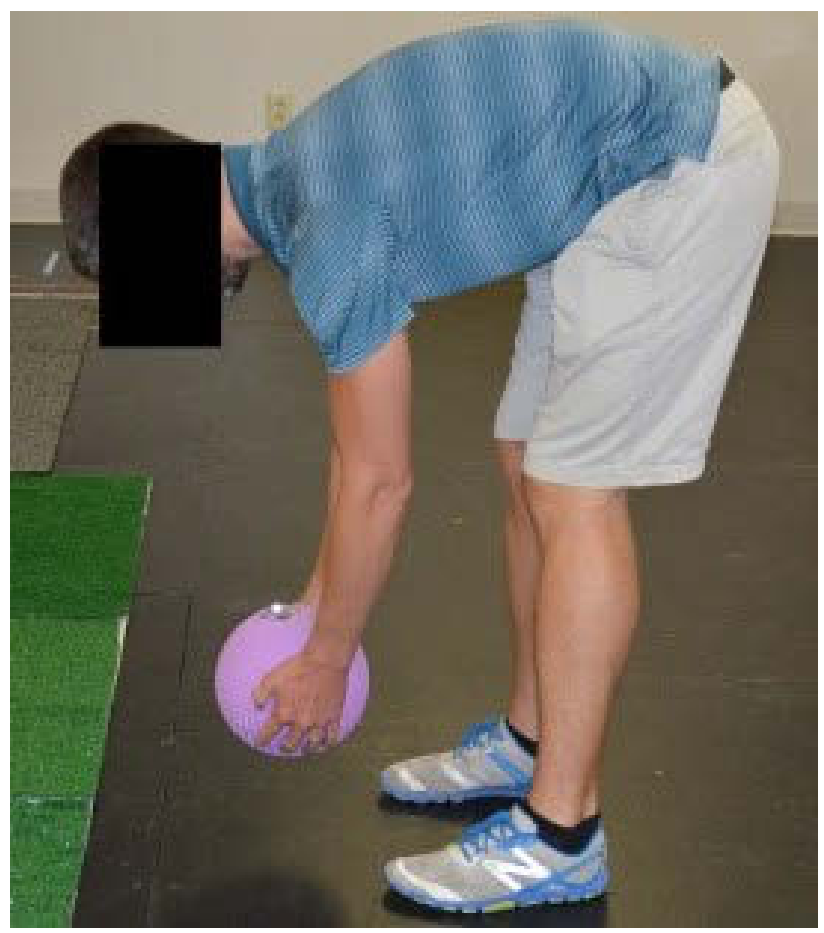

Figure 3-4: Subject performing ball pick up 


\section{Data Analysis}

The EOS scans were used a Le Bonheur technician to generate a 3D reconstruction of the spine and pelvis using SterEOS software. A report was then generated from the reconstruction which contains the L1/L5 lumbar lordosis. If reconstruction was not able to be generated due to a missing key landmark or spine abnormality, a clinical measurement of L1/L5 LL was taken on the sagittal scan using SterEOS. This was taken in the same manner as described before, by measuring the angle created by the lines drawn along the superior endplate of L1 and inferior endplate of L5. SterEOS was also used to generate the marker based LL by measuring the acute angle formed by the lines connecting the screw tips of T12 to L2 and S2 to L4. Both measurements were taken three times on each scan and averaged to reduce the effect of human error. The difference between the anatomical LL and the marker based LL gave the LL correction constant. A LL correction constant was found for each testing session.

Motion files and a static trial were imported into Visual 3D for processing. Marker position was interpolated over a maximum of 10 frames using a $3^{\text {rd }}$ order polynomial, and low pass filtered at $7 \mathrm{~Hz}$ using a digital $4^{\text {th }}$ order Butterworth filter. The static trial was used to generate a rigid-linked segment model made up of eight geometric shapes. The foot, shank, and thigh of each leg were modeled as cones; and the pelvis and torso were be modeled by right elliptical cylinders. The location ankle and knee joint centers are given by the midpoints of the malleoli and epicondyles, respectively.

A landmark which would serve as CASIS was created between the left and right ASIS's. Likewise, CPSIS was created in between the left and right PSIS's. PT was defined as the angle between the lines connecting CASIS and CPSIS, and the horizontal line through CPSIS. Positive pelvic tilt indicates the anterior side of the pelvis is lower than the posterior side, while negative pelvic tilt indicates the posterior side is lower than the anterior side. Lumbar lordosis (LL) was defined as the acute angle between the lines connecting the markers on T12 to L2 and S2 to L4.

\section{Walking}

PT and LL was measured at right and left heel strikes (HS) for each trial. If the trial contained more than one HS on a side, the HS with better marker visibility was used. Due to marker visibility several trials had HS measurements from only one side, however each subject had measurements from at least 10 heel strikes per foot for each session. Stride length was also measured as the distance between RHS and LHS for each trial. The average PT, LL, and stride length were found for each session. The LL correction factor was added to its corresponding LL measurements to give the corrected LL. The difference between pre and post intervention measurements was found for each participant and averaged. A paired t-test was used to determine statistical significance. 


\section{Sit to Stand}

Events were defined for each trial sitting, standing, and maximum force measured by the force plates during lift off. Maximum force always occurred immediately after lift off from the bench. PT and LL was found at each event and the values were averaged for each participant. Corrected LL was found at standing and sitting by adding the correction constant to the marker based LL. A corrected LL for liftoff was not calculated because it was determined that LL at those points was too different from standing for the correction constant to remain valid. The difference between pre and post intervention measurements at sitting, standing and lift off were found for each subject and averaged. A paired t-test was used to determine statistical significance.

\section{Forward Bend}

PT and LL were measured at standing and maximum bend for each trial. Maximum bend was defined as the point at which the marker at $\mathrm{C} 7$ reaches the lowest vertical height. Standing was defined at the point at which $\mathrm{C} 7$ reached the maximum vertical distance. The LL measurements were corrected with the correction constant to give LL*. LL* during maximum bend was not calculated because the LL was too different from standing for the correction constant to remain valid. The difference between pre and post intervention measurements at standing and bending were found for each subject and averaged. A paired t-test was used to determine statistical significance.

\section{Ball Pick Up}

PT and LL was measured at standing and ball pick up. Standing was defined as the maximum height of $\mathrm{C} 7$ during cycles the subject was not holding the ball. To determine how the pattern of motion changes for picking up an object the contribution of pelvic flexion and lumbar flexion was measured. To determine this, pelvic and lumbar flexion was referenced to the standing PT and LL. Pelvic flexion (PF) was found by subtracting the standing PT measurement from the PT measurement for the rest of the cycle (i.e. pelvic flexion at standing is set to zero and positive pelvic flexion indicates bending forward at the pelvis). Lumbar flexion (LF) was found similarly however the negative of the difference was used so positive lumbar flexion indicated bending forward. Total torso flexion (TF) was found by adding the lumbar flexion and pelvic flexion.

$$
\begin{gathered}
\mathrm{PF}=\mathrm{PT}-\mathrm{PT}_{\text {Standing }} \\
\mathrm{LF}=-\left(\mathrm{LL}-\mathrm{LL}_{\text {Standing }}\right) \\
\mathrm{TF}=\mathrm{PF}+\mathrm{LF}
\end{gathered}
$$

Events were then defined at standing, and maximum flexion. Events were also defined at $30^{\circ}$ total flexion, $60^{\circ}$ total flexion, and the moment when the knees bent to determine how the motion changed at different points in the task. The PT, LL, pelvic flexion, and 
lumbar flexion were found at all events. The difference between pre and post intervention measurements was found for each subject and averaged. A paired t-test was used to determine statistical significance at each event.

\section{Results}

Nine participants completed the stretching protocol and follow up testing. One participant dropped out from the study before stretching began. The other two participants were unable to make it back for testing due to scheduling conflicts. All subjects decreased popliteal angle with a mean change of $-18.8^{\circ} \pm 11.6^{\circ}$. The motion capture system was calibrated to a standard deviation below $0.45 \mathrm{~mm}$ for all sessions. This corresponds to a maximum error of $\pm 0.2^{\circ}$ and $\pm 0.8^{\circ}$ for pelvic tilt and lumbar lordosis respectively. EOS scans were taken for all subjects; however a SterEOS reconstruction could not be made for one participant due to a landmark being covered by a calibration ball.

\section{Walking}

Pelvic tilt at heel strike increased for 8 of 9 subjects resulting in a mean change of $2.2^{\circ} \pm 1.2^{\circ}(\mathrm{p}<0.05)$. The change in PT did not correspond to an increase in corrected LL. Stride length also increased for seven subjects, resulting in a mean change of $1.8 \mathrm{~cm}$ $\pm 1.9 \mathrm{~cm}(\mathrm{p}<0.05)$. The results for walking are shown in Table 3-2; all results are in degrees, unless otherwise noted.

\section{Sit to Stand}

There were no significant findings in the sit to stand activity. There was an average increase in PT of $2.1^{\circ} \pm 3.1^{\circ}$ in standing, $0.8^{\circ} \pm 7.6^{\circ}$ in sitting, and $3.0^{\circ} \pm 8.3^{\circ}$ at lift off; however neither was statistically significant. Corrected LL was found in sitting and standing resulting in an average change of $-3.7^{\circ} \pm 9.2^{\circ}$ and $0.8^{\circ} \pm 7.6^{\circ}$ respectively, neither of which were significant. Corrected LL was not found at lift off because the position deviated too far from the position in the EOS scan. The results for sit to stand are shown below in Table 3-3; all results are in degrees.

\section{Forward Bend}

All subjects had an increase in PT while in maximum flexion with a mean increase of $10.6^{\circ} \pm 5.4^{\circ}(\mathrm{p}<0.01)$. PT also increased in standing with a mean change of $4.4^{\circ} \pm 2.8^{\circ}(\mathrm{p}<0.01)$. Corrected LL was found in the standing position, and found to have a mean change of $7.0^{\circ} \pm 7.2^{\circ}(\mathrm{p}<0.05)$. The vertical distance from $\mathrm{C} 7$ to the floor also decreased by $7.5 \mathrm{~cm} \pm 6.9 \mathrm{~cm}(\mathrm{p}<0.05)$, indicating a greater range of flexion. The results are shown in Table 3-4 in units of degrees, unless otherwise noted. 
Table 3-2: Change in popliteal angle (PA), pelvic tilt (PT), corrected lumbar lordosis (LL) and stride length (SL) during gait

\begin{tabular}{lcccc}
\hline Subject & PA & PT & LL & SL (cm) \\
\hline 145 & -12.5 & 1.1 & -0.5 & 0.6 \\
148 & -22.5 & 3.9 & 16.1 & 2.6 \\
150 & -22 & 3.0 & -4.5 & 3.4 \\
151 & -27.5 & 4.8 & -0.9 & -0.3 \\
152 & -39 & -4.0 & -6.6 & 2.4 \\
153 & -15 & 3.6 & -5.3 & 3.1 \\
155 & -5 & 2.3 & 2.8 & 4.8 \\
156 & -1.5 & 1.5 & 3.8 & -0.9 \\
158 & -24.5 & 2.7 & 6.3 & 0.2 \\
Mean & $\mathbf{- 1 8 . 8}$ & $\mathbf{2 . 1}$ & $\mathbf{1 . 2}$ & $\mathbf{1 . 8}$ \\
St. Dev & $\mathbf{1 1 . 6}$ & $\mathbf{2 . 6}$ & $\mathbf{7 . 1}$ & $\mathbf{1 . 9}$ \\
p-value & $\mathbf{0 . 0 0 1}$ & $\mathbf{0 . 0 4}$ & $\mathbf{0 . 6 1}$ & $\mathbf{0 . 0 3}$ \\
\hline
\end{tabular}

Table 3-3: Change in PT and corrected LL for each participant during sit-tostand

\begin{tabular}{lcccccc}
\hline & \multicolumn{3}{c}{ Anterior Pelvic Tilt } & & \multicolumn{2}{c}{$\begin{array}{c}\text { Corrected Lumbar } \\
\text { Lordosis }\end{array}$} \\
\cline { 2 - 4 } \cline { 6 - 7 } Subject & Sitting & Lift Off & Standing & & Sitting & Standing \\
\hline 145 & -3.7 & -6.1 & -1.5 & & -0.5 & 0.7 \\
148 & 2.2 & -0.4 & -1.5 & & 14.9 & 13.0 \\
150 & -13.7 & -7.2 & 4.8 & & -8.3 & -17.3 \\
151 & 7.2 & 5.7 & 0.3 & & -2.8 & 3.9 \\
152 & -4.0 & 2.2 & 5.1 & & -8.7 & -6.6 \\
153 & 8.4 & 6.9 & -2.6 & & -2.8 & -3.5 \\
155 & 4.5 & 18.7 & 3.7 & & $4.4 *$ & $4.7 *$ \\
156 & -4.0 & -3.0 & 2.9 & & 4.8 & -4.1 \\
158 & 6.2 & 10.1 & 0.5 & & 6.3 & -7.0 \\
Mean & 0.8 & 3.0 & 2.1 & & -3.7 & 0.8 \\
St. Dev & 7.6 & 8.3 & 3.1 & & 9.2 & 7.6 \\
p-value & 0.76 & 0.31 & 0.07 & & 0.29 & 0.75 \\
\hline
\end{tabular}

*LL correction constant calculated from clinical measurements based off of radiograph 
Table 3-4: Change in PT, corrected LL, and $\mathrm{C} 7$ height during forward bend

\begin{tabular}{lccccc}
\hline & \multicolumn{2}{c}{ Standing } & & \multicolumn{2}{c}{ Max Flexion } \\
\cline { 2 - 3 } \cline { 5 - 6 } Subject & PT & Corrected LL & & PT & C7 Height (cm) \\
\hline 145 & 3.1 & 4.2 & & 2.2 & -3.1 \\
148 & 2.9 & 20.4 & & 3.1 & 0.5 \\
150 & 6.9 & 4.6 & & 11.0 & -11.8 \\
151 & 9.1 & 7.5 & & 19.6 & -11.8 \\
152 & 3.2 & -1.2 & & 9.9 & -15.1 \\
153 & 5.6 & -0.9 & & 11.8 & -4.7 \\
155 & 3.9 & $7.3 *$ & & 15.0 & -9.4 \\
156 & -0.6 & 5.0 & & 12.9 & -15.9 \\
158 & 5.6 & 16.1 & & 9.8 & 3.4 \\
Mean & $\mathbf{4 . 4}$ & $\mathbf{7 . 0}$ & & $\mathbf{1 0 . 6}$ & $\mathbf{- 7 . 5}$ \\
St. Dev & $\mathbf{2 . 8}$ & $\mathbf{7 . 2}$ & & $\mathbf{5 . 4}$ & $\mathbf{6 . 9}$ \\
p-value & $\mathbf{0 . 0 0 1}$ & $\mathbf{0 . 0 2}$ & $\mathbf{0 . 0 0 0 4}$ & $\mathbf{0 . 0 1}$ \\
\hline *LL correction constant calculated from clinical measurements based off of radiograph
\end{tabular}




\section{Ball Pick Up}

Subjects had a mean increase of $2.0^{\circ} \pm 2.6(\mathrm{p}=0.05)$, at standing $11.1^{\circ} \pm 12.4^{\circ}$ $(\mathrm{p}<0.05)$ at knee bend, and $7.0^{\circ} \pm 9.1^{\circ}(\mathrm{p}<0.05)$ during maximum flexion. There was also an increase of corrected lumbar lordosis at standing, but this increase was not statistically significant. Results are shown in Table 3-5; all measurements are in degrees.

Subjects showed increased lumbar flexion at $30^{\circ}$ and $60^{\circ}$ of total flexion, and while the results are significant the difference is mostly a result of the increased PT at standing. Results are shown in Table 3-6; all results are in degrees.

\section{Discussion}

All participants increased in hamstring flexibility, which is represented by a decrease in popliteal angle, however some participants had much greater gains than others. Subject 156 had the smallest gain in hamstring length and was the only subject whose popliteal angle remained greater than 25 degrees. This suggests that the stretching protocol was more effective for some participants than others. This may be due to participant compliance or perhaps some subjects had a greater predisposition to tight hamstrings that might require a longer intervention. All subjects showed some increase in hamstring length, therefore they were all still included for the analysis.

\section{Walking}

Eight subjects showed an increase in anterior pelvic tilt at heal strike; however this did not correlate to an increase in lumbar lordosis. While it has been reported that increased PT while standing would cause an increase in LL [10], the relationship between the PT and LL during gait proves to be more complicated. While lengthening the hamstrings does appear to alter the positioning of the pelvis, that doesn't necessarily mean the lumbar spine will follow. This may be due to movement within the SI joint or the influence of other muscles including the abdominal, erector spinae, and psoas major which also work to stabilize the lumbar spine.

Several authors report an out of phase relationship between PT and LL within the gait cycle although this relationship was highly variable within each subject [30]. While PT tended to have consistent peaks and valleys within the gait cycle across participants, LL would vary considerably between participants. Rowe and White and Crosbie et al. both also noted high variability of LL patterns between subjects during gait [31,32]. These results were very similar to what was found in this study. While some subjects appeared to have an in phase relationship between LL and PT within the gait cycle, others had the opposite, or no relationship at all. To complicate the matter further, due to the amount of movement present in the four vertebrae; the LL plot can be rather noisy throughout the gait cycle. Standard deviation of the LL measurements for each subject can range from $0.5^{\circ}$ to $1.5^{\circ}$, which introduces quite a bit of variability given the average 
Table 3-5: Change in PT and corrected LL during ball pick up

\begin{tabular}{|c|c|c|c|c|c|c|}
\hline \multirow[b]{2}{*}{ Subject } & \multicolumn{5}{|c|}{ Pelvic Tilt } & \multirow[b]{2}{*}{$\begin{array}{c}\text { Standing } \\
\text { LL }\end{array}$} \\
\hline & Standing & $30^{\circ}$ & $60^{\circ}$ & $\begin{array}{c}\text { At Knee } \\
\text { Bend }\end{array}$ & $\begin{array}{c}\text { Max } \\
\text { Flexion }\end{array}$ & \\
\hline 145 & 0.9 & 0.5 & -1.1 & 3.5 & 1.0 & 3.7 \\
\hline 148 & 4.4 & 2.9 & 1.6 & -6.3 & -1.9 & 20.7 \\
\hline 150 & 3.3 & 2.3 & 0.4 & 18.2 & -5.0 & -9.5 \\
\hline 151 & 5.2 & 7.5 & 6.6 & 30.7 & 9.3 & 4.0 \\
\hline 152 & -1.9 & 0.6 & 3.0 & -2.9 & 5.0 & -3.3 \\
\hline 153 & 1.8 & 3.3 & 3.0 & 21.8 & 7.1 & -0.2 \\
\hline 155 & 2.1 & 1.5 & 0.7 & 9.3 & 21.7 & $6.5^{*}$ \\
\hline 156 & -1.8 & -0.5 & -0.8 & 5.2 & 6.0 & 7.8 \\
\hline 158 & 3.9 & -6.8 & -4.1 & 20.2 & 20.1 & 11.7 \\
\hline Mean & 2.0 & 1.3 & 1.0 & 11.1 & 7.0 & 4.6 \\
\hline St. Dev & 2.6 & 3.8 & 3.1 & 12.4 & 9.1 & 8.7 \\
\hline p-value & 0.05 & 0.35 & 0.35 & 0.03 & 0.048 & 0.15 \\
\hline
\end{tabular}

* LL correction constant calculated from clinical measurements based off of radiograph

Table 3-6: Change in pelvic flexion and lumbar flexion during ball pick up

\begin{tabular}{lccccc}
\hline & \multicolumn{2}{c}{$\mathbf{3 0}^{\circ}$ Total Flexion } & & \multicolumn{2}{c}{$\mathbf{6 0}^{\circ}$ Total Flexion } \\
\cline { 2 - 3 } \cline { 5 - 6 } Subject & $\begin{array}{c}\text { Pelvic } \\
\text { Flexion }\end{array}$ & $\begin{array}{c}\text { Lumbar } \\
\text { Flexion }\end{array}$ & & $\begin{array}{c}\text { Pelvic } \\
\text { Flexion }\end{array}$ & $\begin{array}{c}\text { Lumbar } \\
\text { Flexion }\end{array}$ \\
\hline 145 & 0.0 & 0.0 & & -1.6 & 1.9 \\
148 & -1.4 & 1.7 & & -2.8 & 3.1 \\
150 & -1.4 & 1.2 & & -3.2 & 3.5 \\
151 & -0.5 & 0.5 & & -1.5 & 1.6 \\
152 & -0.7 & 0.8 & & 1.7 & -1.5 \\
153 & 0.6 & -0.7 & & 0.3 & 0.0 \\
155 & -0.9 & 0.6 & & -1.6 & 1.9 \\
156 & -1.0 & 1.5 & & -1.2 & 1.4 \\
158 & -10.0 & 9.9 & & -7.3 & 7.6 \\
Mean & $\mathbf{- 1 . 7}$ & $\mathbf{1 . 7}$ & & $\mathbf{- 1 . 9}$ & $\mathbf{2 . 2}$ \\
St. Dev & $\mathbf{3 . 2}$ & $\mathbf{3 . 1 5}$ & & $\mathbf{2 . 5}$ & $\mathbf{2 . 5}$ \\
p-value & $\mathbf{0 . 1 5}$ & $\mathbf{0 . 1 4}$ & & $\mathbf{0 . 0 5}$ & $\mathbf{0 . 0 3}$ \\
\hline
\end{tabular}


change was only $1.2^{\circ}$. Given the variability of LL, it would make it very difficult to find a positive correlation between anterior PT and LL.

Another limitation to the study is the accuracy of the LL measurement and the LL correction method. The motion capture system has a $\pm 0.5 \mathrm{~mm}$ marker error which would result in a LL error of approximately $\pm 0.8^{\circ}$. When added to the average error in the LL correction method of $3.2^{\circ}$, this results in a $4^{\circ}$ error in the corrected LL. Given the relatively small change measured in LL, if there is a LL change caused by increased PT, the current system is likely not accurate enough to detect the change.

Despite the inconclusive LL results, stretching hamstrings did achieve a positive change in PT during gait as well as increase the average stride length. Taking only the subjects with a positive increase in PT, the average change becomes $2.9^{\circ}$. While this is still a relatively small change, it may become more significant if more participants are used who achieve a greater improvement in popliteal angle. These results are promising for understanding the effect of tight hamstrings on pelvic kinematics during gait, and to the authors knowledge no other study has utilized a hamstring stretching intervention to determine its effect on gait. The relationship between LL and PT during gait and its connection to LBP remains a subject for future studies.

\section{Sit to Stand}

None of the changes measured during the sit to stand were of statistical significance. Although there was a mean increase in PT at all events within the cycle, the large standard deviation prevented any results to be statistically significant. It is possible that hamstring length does not have any effect during sit to stand, however there are some limitations that should be considered for these results. One great contributor to the large standard deviation could be lack of consistency between subjects. Subjects were instructed not to use their arms during standing; however arm placement was not specified. This resulted in some subjects keeping their arms down at their side, out in front, or even crossed over the chest. Unfortunately, the arm position was not noted during the first testing so there was no way to ensure the same arm position was used during the follow up. The arm placement would significantly alter the momentum of the upper body which would change the lumbar and pelvic kinematics. A future study should provide more specific instructions on where to place hands throughout the cycle.

\section{Forward Bend}

It was not surprising that anterior PT increased significantly during maximum flexion of forward bend. All subjects experienced an increase, and this activity showed the greatest increase in PT out of all the activities. These results agree with several other studies including Gajdosik et al. and Li et al $[9,27]$. Unfortunately, due to the degree of lumbar flexion, the LL correction was not able to be used beyond standing position; therefore a comparison of LL was not made. There was also an increase of LL and PT at 
standing. This strengthens the argument that there is a link between LL and PT during standing which is in agreement with the findings of Day et al [10], however the results should be taken with caution. The standard deviation of the change in LL is greater than the average change measured. In general, participants who had a greater change in PT tended to have greater increases in LL, however a clear correlation did not exist, and for some this relationship was not true. Subjects 152 and 153 both had an increase in PT, but a decrease in LL, and subject 156 showed a decrease in PT but still had an increase in LL. It is also important to consider that the LL changes for 152 and 153 are within the error of measurement; however the error of measurement should also be considered for the other results. With a LL error of approximately $4^{\circ}$, using a more accurate method of measurement could strengthen or weaken the correlation between PT and LL while standing.

Another limitation was that the standing measurement was taken while the subject was still in motion since the subject went straight into extension from the bend. Standing was defined by the highest vertical distance of $\mathrm{C} 7$, which allowed it to be measured consistently every repetition, however the subject did not settle into a natural standing pose. During ball pick up subjects were required to pause for two seconds, which gives better representation of a normal standing pose.

\section{Ball Pick Up}

Ball pick up is a very similar motion to forward bend therefore it was expected that the results would be similar, and for the most part they were similar. There was an increase in PT at maximum flexion and standing, and while there was an average increase in LL in standing, this increase was not statistically significant. However the PT at maximum flexion was much more variable at ball pick up than forward bend and therefore resulted in a smaller, less significant change in PT. It was expected that participants change in PT at ball pick up would also match their change in PT during forward bend, however this was not the case. Two participants actually had a decrease in PT during ball pick up whereas all participants had an increase in PT for forward bend.

While these activities are similar they have some major differences which should be considered. Ball pick up requires the subject to bend to a position where they could pick up the ball. To accomplish this most participants, especially during the baseline testing, had to bend their knees. Once the knees are bent it brings a lot of variability into the pelvic positioning, in general allowing for more pelvic tilt. During the follow up testing some participants still had to bend their knees, however others did not which made it difficult to compare the two measurements.

Due to the variability knee bending introduces, the PT was also found the moment before the subject bent their knees. Unfortunately, finding the point that the knees began to flex was somewhat subjective. Some subjects maintained perfectly straight knees up until the point they could not bend any further creating a distinct point of knee flexion. However, others anticipated having to bend their knees and either started with a small 
amount of knee flexion or allowed a small amount of knee flexion to creep in during early phases of torso flexion. The small amount of knee flexion was only visible after viewing the motion files, therefore could not be corrected during testing. For all subjects, there was a point at which there was a sharp increase in knee flexion which is the moment that was used for the knee bend measurement. For most subjects this was the corresponded to the time at which the knee first began to flex, however several had already had a small amount of flexion which increases variability of the results. Despite the variability, the average increase in PT of $11.1^{\circ}$ was closer to the forward bend average. Individual changes were also more consistent, despite a few outliers. For example subject 148 had a decrease in PT of 6 degrees, however after closer examination it was found that this subject started with a small amount of knee flexion at the beginning of the task.

The change in lumbar flexion and pelvic flexion at $30^{\circ}$ and $60^{\circ}$ also gave insight on whether the pattern of motion had changed during ball pick up. One study reported greater lumbar flexion in subjects with a history of LBP during the first phase of forward bend. They also noted that many of the LBP subjects had tight hamstrings, which may have contributed to increased lumbar flexion [33]. It was thought that after stretching participants may utilize the pelvis more during the initial phases of flexion, however that is not what the results showed. It appears that lumbar flexion actually increased and pelvic flexion decreased, however the small change detected could be an artifact of the different reference PT and LL taken at standing. Looking at the PT at $30^{\circ}$, it appears that even though participants start at a more anteriorly tilted pelvis, they do not initiate pelvic flexion any sooner when bending forward. Even at $60^{\circ}$ flexion, most participants are still not utilizing the increased pelvis range of motion they have acquired through hamstring stretching. The increased range of motion is only utilized later in the bend which is shown by the increased PT at knee bend and at maximum flexion. This is an important observation, as even though their pelvic range of motion has changed, their behavior has remained relatively the same.

Due to the two second pause and consistent hand positioning at standing, the ball pick up was arguably the most credible activity to determine the effect of tight hamstrings on standing posture. The results showed an average increase in PT of $2.0^{\circ}$ which was slightly less than the increase during the standing position at forward bend. There was also an average increase in LL of 4.6 however this was not statistically significant. Just like in forward bend, participants with a greater change in PT tended to have increased LL; however there was still not a strong correlation.

\section{Conclusion}

The hamstring stretching protocol was effective at increasing hamstring length for all participants, and LL correction method allowed a more accurate comparison of LL measured from two separate sessions. The effect of hamstring stretching was shown to increase anterior PT at heal strike during gait; however the effect this has on LL is still inconclusive. PT also increased during standing for the ball pick up and forward bend. 
Subjects also saw a significant increase in LL at standing during the forward bend, and although there was an average increase in LL at standing for ball pick up, the change was not significant. These results strengthen the argument that increased PT will cause an increase in LL, however due the accuracy of the correction method, large variation in results, and low sample size; future work is needed to make this conclusion. The largest change in PT was found at maximum flexion during ball pick up and forward bend. Despite the increased pelvic range of motion, the behavior while picking up an object did not change, and the extra range of motion was only utilized at later phases of flexion. 


\section{CHAPTER 4. GENERAL DISCUSSION}

The goal of this thesis was two-fold; first was to develop and validate a method that allows the comparison of gait lab LL measurements taken from different sessions. The second was to determine the effect that hamstring lengthening has on pelvic tilt and lumbar lordosis during gait and other activities. The first was accomplished by using EOS Bi-planar X-ray scans of twenty-one subjects in two different poses to compare two different methods of LL correction. The most accurate method utilized a correction constant was found to have an average error of $3.2^{\circ}$ or $6.2 \%$. Despite the error, it still allowed a more accurate comparison of LL measurements taken from multiple sessions.

Utilization of the lumbar correction constant allows a more accurate comparison of LL measurements taken in the gait lab. While the EOS Bi-Planar X-Ray system may not be accessible to many gait labs; using a single sagittal X-ray is a viable alternative. There is a certain amount of error introduced by the correction constant; however this error is far less than the error due to inaccurate marker placement. Use of this correction method could allow clinical gait labs to measure treatment efficacy for spine abnormalities by allowing the comparison of spinal measurements from different sessions. While this method was used only on the lumbar region, there is no reason a similar approach couldn't be used to measure kyphosis or curves in other planes.

The second goal was accomplished by having nine subjects with clinically tight hamstrings perform walking, sit to stand, forward bend, and picking up an object while being recorded in a motion capture laboratory. After which the subjects completed a six week stretching protocol, and then returned to the motion capture lab to perform the same activities. LL measurements were corrected using the correction constant and the change in PT and corrected LL was found for each activity. All subjects increased their hamstring length which resulted in an increase in PT during walking at heal strike, forward bend while standing and bending, and picking up a ball at standing and ball pick up. The increase in PT did not necessarily correspond to an increase in PT. An increased LL was seen at standing, but this was only statistically significant during forward bend. The greatest increase in PT was seen during bending activities.

The results of this study suggest that a person with tight hamstrings will tend to tilt their pelvis more posteriorly during gait. Even though it is still unclear how that affects LL during gait, it is still important to consider the other potential effects of changing PT. Other studies have reported that increased anterior PT causes an increase in acetabular coverage on the femoral head [34]. This would theoretically increase the load bearing surface area, resulting in a decreased load per unit area on the hip. Decreased load per unit area may decrease the risk of osteoarthritis in the hip [35]. The increase in LL seen at standing did support the link between LL and PT, and there are several studies which also support this link $[10,36,37]$. Boulay et al reported that decreased pelvic incidence, which is related to pelvic tilt, will affect the sagittal balance of the entire spine. He found that subjects with lower pelvic incidence experienced a flattened spinal curve characterized by decreased LL and thoracic kyphosis. Though the 
change in lordosis was small, it has been shown that even small changes can result in significant changes in force on the spine. Srbinoska et al found that even though larger loads aren't always associated with a change in LL, just a $10^{\circ}$ decrease in LL could result in an increased force of $200 \mathrm{~N}$ on the vertebral body [13].

The largest increase in PT was seen during bending activities. It was not possible to accurately measure change in LL at this point, the increased range of PT will allow participants to perform tasks at lower heights with less lumbar flexion. Loaded lumbar flexion squeezes the nucleus pulposus posteriorly which creates greater tensile stress on the posterior portion of the annulus [15]. One study reported that the intradiscal pressure while holding an $8 \mathrm{~kg}$ weight at $30^{\circ}$ flexion can be six times as high as standing intradiscal pressure [38]. The pressure can be great enough to allow fluid from the nucleus to squeeze out causing a herniated disc [15]. It is this reason that lumbar flexion should be minimalized when lifting an object. Even though the increased range of PT was not utilized until the later phases of bending, if a subject is aware of the behavior they could potentially utilize more pelvic motion to avoid lumbar flexion.

\section{Limitations}

While the LL correction method allowed comparison of gait lab measured LL, it still had many limitations. The first being the limited range of LL which the correction constant can be accurately applied. The method needs to be validated against greater ranges of lumbar flexion to determine whether it can be used for activities where lumbar flexion greatly differs from the radiograph. Because of this the correction method was only used on measurements taking in a standing or sitting position.

Another limitation of the correction method is that any rotation of the subject was not accounted for in the marker LL measurements on the sagittal scan. Since EOS uses a bi-planar x-ray it would be possible to find the three dimensional points of the markers and calculate a true angle in three dimensional space, however it is not a current capability of the software. Therefore, measurements were taken assuming the scan accurately represents the sagittal plane of the subject.

A major limitation of the hamstring study was the small sample size of the study. Although 12 were initially enrolled in the study, only 9 completed the post intervention follow up testing. Furthermore, several participants did not achieve the hamstring flexibility that was desired, and one subject still had a popliteal angle greater than $25^{\circ}$. The error in the corrected LL also creates another limitation to this study. The combined error of the motion capture system and the correction constant is about $4^{\circ}$, which is also greater than the small changes seen during activities such as walking. 


\section{Future Work}

Further studies should continue to improve on the LL correction method including validation at greater degrees of lumbar flexion. Accuracy may improve by measuring the marker based angle in three dimensions as mentioned earlier. However even with those improvements, it still might not achieve the desired accuracy. It is worth investigating using all the markers on the lumbar region and fitting a curve to all the points. This approach may allow for a more accurate comparison of lumbar lordosis between testing, however it would be more difficult to relate the values to clinical measurements.

The greatest limitation of the hamstring lengthening study is the small sample size; therefore continued recruitment of subjects would greatly increase the strength of this study. It may be beneficial to also require a minimum improvement of hamstring flexibility before the follow up testing, which may result in more substantial changes in pelvic and lumbar kinematics. There are also countless other measures that can be investigated including the kinetics of the pelvis and lumbar spine. To strengthen the connection to lower back pain, the next phase of this study should include a cohort of subjects with tight hamstrings and a history of LBP. 


\section{LIST OF REFERENCES}

1. $\quad$ Balague, F., et al., Non-specific low back pain. Lancet, 2012. 379.

2. Luo, X., et al., Estimates and patterns of direct health care expenditures among individuals with back pain in the United States. Spine, 2004. 29(1).

3. Guo, H.-R., et al., Back Pain Prevalence in the US Industry and Esitmates of Lost Workdays. American Journal of Public Health, 1999. 89(7): p. 1029-1035.

4. Chou, R., et al., Diagnosis and Treatement of Low Back Pain: A Joint Clinical Practice Guidline from the American College of Physicians and the American Pain Society. Annuals of Internal Medicine, 2007. 147(7).

5. Ullrich, P.F. Speific Hamstring Stretches for Back Pain Relief. 2009 5/24/2016]; Available from: http://www.spine-health.com/wellness/exercise/specifichamstring-stretches-back-pain-relief.

6. Sherman, K., et al., Mediators of Yoga and Stretching for Chronic Low Back Pain. Evidence-Based Complementary and Alternative Medicine, 2013. 2013.

7. Arab, A.M. and M. Reza, Hamstring muscle length and lumbar lordosis in subjects with different lifestyle and work setting: Comparison between individuals with and without chronic low back pain. Journal of Back and Musculoskeletal Rehabilitation, 2013. 27(1): p. 63-70.

8. Radwan, A., et al., Evaluation of intra-subject difference in hamstring flexibility in patients with low back pain: An exploratory study. Journal of Back and Musculoskeletal Rehabilitation, 2014. 28(1).

9. Li, Y., P. McClure, and N. Pratt, Effect of Hamstring Muscle Stretching on Standing Posture and on Lumbar and Hip Motions During Forward Bending. Physical Therapy, 1996. 76(8).

10. Day, J., G. Smidt, and T. Lehmann, Effect of Pelvic Tilt on Standing Posture. Physical Therapy, 1984. 64(4).

11. Adams, M., A.F. Mannion, and P. Dolan, Personal Risk Factors for First-Time Low Back Pain. Spine, 1999. 24(23): p. 2497.

12. Keller, T., et al., Influence of spine morphology on intervertebral disc loads and stresses in aymptomatic adults: implications for the ideal spine. Spine Journal, 2005. 5(3).

13. Srbinoska, H., et al., Correlation between back shape and spinal loads. Journal of Biomechancs, 2013. 46: p. 1972-1975.

14. Lopez-Minarro, P., et al., Acute Effects of Hamstring Stretching on Sagittal Spinal Curvatures and Pelvic Tilt. Journal of Human Kinetics, 2012. 31.

15. Calais-Germain, B., Anatomy of Movement. 1993, Seattle, WA: Eastland Press, Inc.

16. Frankel, M.N.V.H., Basic Biomechanics of the Musculoskeletal System. Third ed. 2001, New York, New York: Lippincott Williams \& Wilkins.

17. Rah, P.P., Intervertebral Disc: Anatomy-Pysiology-Pathophysiology-Treatment. World Institute of Pain, 2007. 8(1): p. 18-44.

18. Jeffrey C. Lotz, O.K.C., Jennie Chin, Neil Duncan, Ellen Liebenberg, Compression-Induced Degeneration of the Intervertebral Disc: An in Vivo Mous Model and Finite Element Study. Biomechanics, 1998. 
19. Fischer, S.J. and D.K. Park. Hamstring Muscle Injuries. Ortho Info 2015; Available from: http://orthoinfo.aaos.org/topic.cfm?topic=a00408.

20. Hashemirad, F., et al., Validity and reliability of skin markers for measurement of intersegmental mobility at L2-3 and L3-4 during lateral bending in healthy individuals: A fluoroscopy study. Journal of Bodywork \& Movement Therapies, 2012. 17.

21. Morl, F. and R. Blickhan, Three-Dimensional relation of skin markers to lumbar vertebrae of healthy subjects in different postures measured by open MRI. European Spine Journal, 2006. 15.

22. Illes, T. and S. Somoskeoy, The EOS imaging system and its uses in daily orthopaedic practice. International Orthopaedics, 2012. 36.

23. Assaf, D., Kinematic and Kinetic Effects of Alterations in Lumbar Lordosis in People with Tight Hamstrings, in Department of Biomedical Engineering. 2015, University of Tennessee.

24. Schmid, S., et al., Using Skin Markers for Spinal Curvature Quantification in Main Thoracic Adolescent Idiopathic Scoliosis: An Explorative Radiographic Study. PLOS One, 2015. 10.

25. Whittle, M.W. and D. Levine, Measurement of lumbar lordosis as a component of clinical gait analysis. Gait \& Posture, 1997. 5.

26. Snider, K., et al., Palpatory Accuracy of Lumbar Spinous Processes Using Multiple Bony Landmarks. Journal of Manipulative and Physiological Therapeutics, 2011. 34(5).

27. Gajdosik, R., C. Albert, and J. Mitman, Influence of Hamstring Length on the Standing Position and Flexion Range of Motion of the Pelvic Angle, Lumbar Angle, and Thoracic Angle. Journal of Orthopaedic \& Sports Physical Therapy, 1994. 20(4): p. 213-219.

28. Spernoga, S., et al., Duration of Maintained Hamstring Flexibility After a OneTime, Modified Hold-Relax Stretching Protocol. Journal of Athletic Training, 2001. 36(1): p. 44-48.

29. Whitehead, C.L., et al., The effect of simulated hamstring shortening on gait in normal subjects. Gait \& Posture, 2007. 26: p. 90-96.

30. Whittle, M.W. and D. Levine, Three-dimensional relationships between the movements of the pelvis and lumbar spine during normal gait. Human Movement Science, 1999. 18: p. 681-692.

31. Crosbie, J., R. Vachalathiti, and R. Smith, Patterns of spinal motion during walking. Gait \& Posture, 1995. 5: p. 6-12.

32. Rowe, P.J. and M. White, Three dimensional, lumbar spinal kinematics during gait, following mild musculo-skeletal low back pain in nurses. Gait \& Posture, 1996. 4: p. 242-251.

33. Esola, M., et al., Analysis of Lumbar Spine and Hip Motion During Forward Bending in Subjects With and Without a History of Low Back Pain. SPINE, 1996. 21(1): p. 71-78.

34. Henebry, A. and T. Gaskill, The Effect of Pelvic Tilt on Radiographic Markers of Acetabular Coverage. The American Journal of Sports Medicine, 2013.

35. Bierma-Zeinstra, S. and B. Koes, Risk factors and prognostic factors of hip and knee osteoarthritis. Nature Reviews Rheumatology, 2007. 3: p. 78-85. 
36. Vaz, G., et al., Sagittal morphology and equilibrium of pelvis and spine. European Spine Journal, 2002. 11(1): p. 80-87.

37. Boulay, C., et al., Sagittal alignment of spine and pelvis regulated by pelvic incidence: standard values and prediction of lordosis. European Spine Journal, 2006. 15: p. 415-422.

38. Schultz, A., et al., Loads on the Lumbar Spine. The Journal of Bone and Joint Surgery, 1982. 64(5). 


\section{APPENDIX A. INSTITUTIONAL REVIEW BOARD APPROVAL}

\begin{tabular}{lr}
\hline THE UNIVERSITY OF TENNESSEE \\
Health Science Center \\
\hline \\
Institutional Review Board \\
910 Madison Avenue, Suite 600 \\
Memphis, TN 38163 \\
Tel: (901) 448-4824
\end{tabular}

March 18, 2015

Bill Mihalko, M.D., Ph.D.

UTHSC - COM - Orthopaedic Surgery \& Biomedical Engineering

E226 Coleman College of Medicine Building

956 Court Avenue

Memphis, TN 38163

Re: 15-03692-FB UM

Study Title: Hamstring Tightness and Pelvic Position during Activity

Dear Dr. Mihalko:

The IRB has received your written acceptance of and/or responses dated 03/18/2015 and $3 / 11 / 2015$ to the provisos outlined in our correspondence of $3 / 12 / 2015$ and $3 / 4 / 2015$ concerning the application for the above referenced project. The IRB has reviewed these materials and determined that they comply with proper consideration for the rights and welfare of human subjects and the regulatory requirements for the protection of human subjects. Therefore, this letter constitutes full approval by the IRB of your application Version 1.3 including:

- Recruitment script, dated 3/2/2015 (stamped IRB approved 3/18/2015)

- Main consent form, dated 3/10/2015 (stamped IRB approved 3/18/2015)

The UTHSC IRB stamped-approved consent form must be used to enroll prospective subjects in the study.

Approval of this study will be valid from $3 / 18 / 2015$ to 3/4/2016.

The IRB has determined that the informed consent form, incorporating the authorization of subjects to use their protected health information in research, complies with the federal privacy regulations as specified in 45 CFR 160 and 45 CFR 164. 
In the event that subjects are to be recruited using solicitation materials, such as brochures, posters, web-based advertisements, etc., these materials must receive prior approval of the IRB. Any revisions in the approved application must also be submitted to and approved by the IRB prior to implementation. In addition, you are responsible for reporting any unanticipated serious adverse events or other problems involving risks to subjects or others in the manner required by the local IRB policy.

Finally, re-approval of your project is required by the IRB in accord with the conditions specified above. You may not continue the research study beyond the time or other limits specified unless you obtain prior written approval of the IRB.

Sincerely,

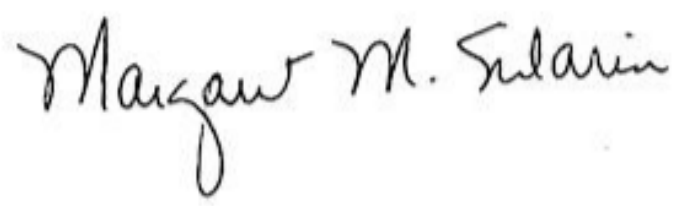

Signature applied by Margaret M Sularin on 03/18/2015 01:44:54 PM CDT

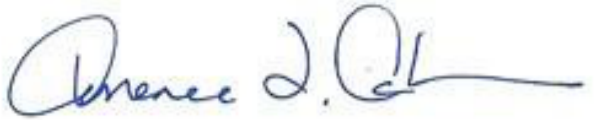

Signature applied by Terrence F Ackerman on 03/18/2015 01:45:37 PM CDT

Margaret M. Sularin, LMSW, RDN, CCRP, CIM, CIP Senior Regulatory Specialist UTHSC IRB
Terrence F. Ackerman, Ph.D.

Chairman

UTHSC IRB 


\section{APPENDIX B. ADDITIONAL GRAPHS}

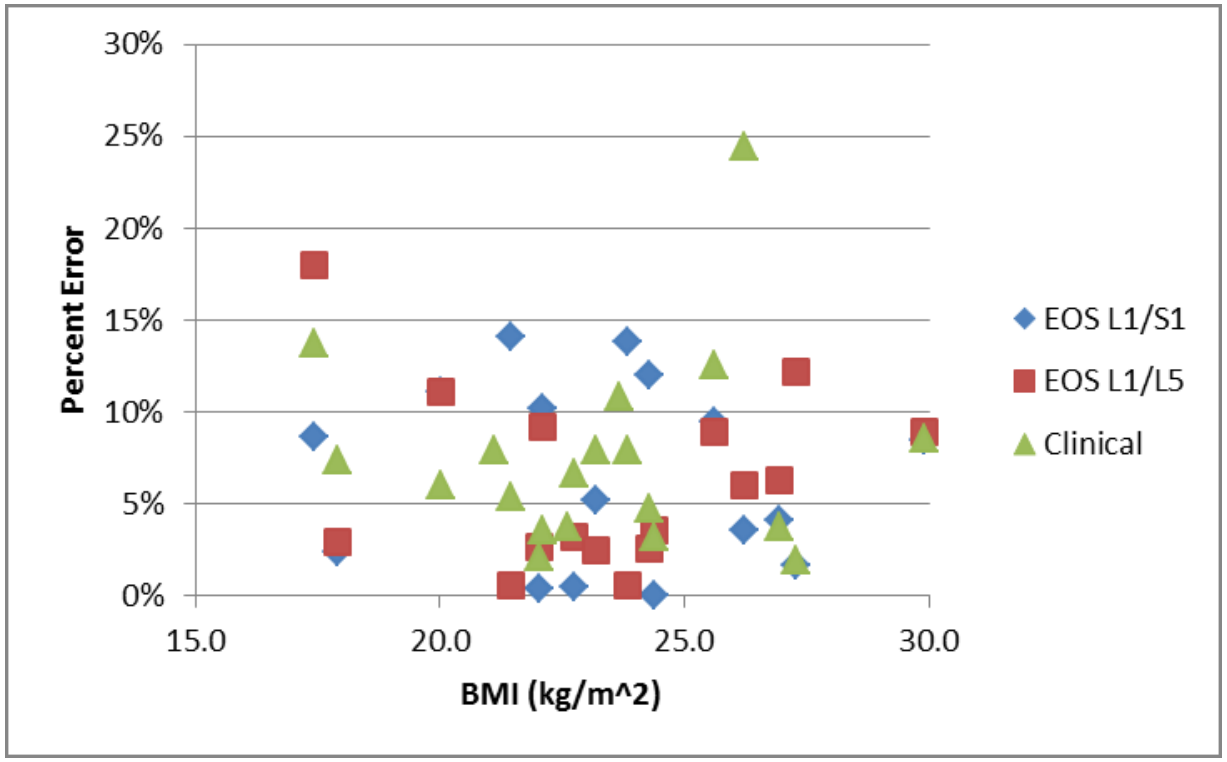

Figure B-1: Percent error vs BMI using the difference method

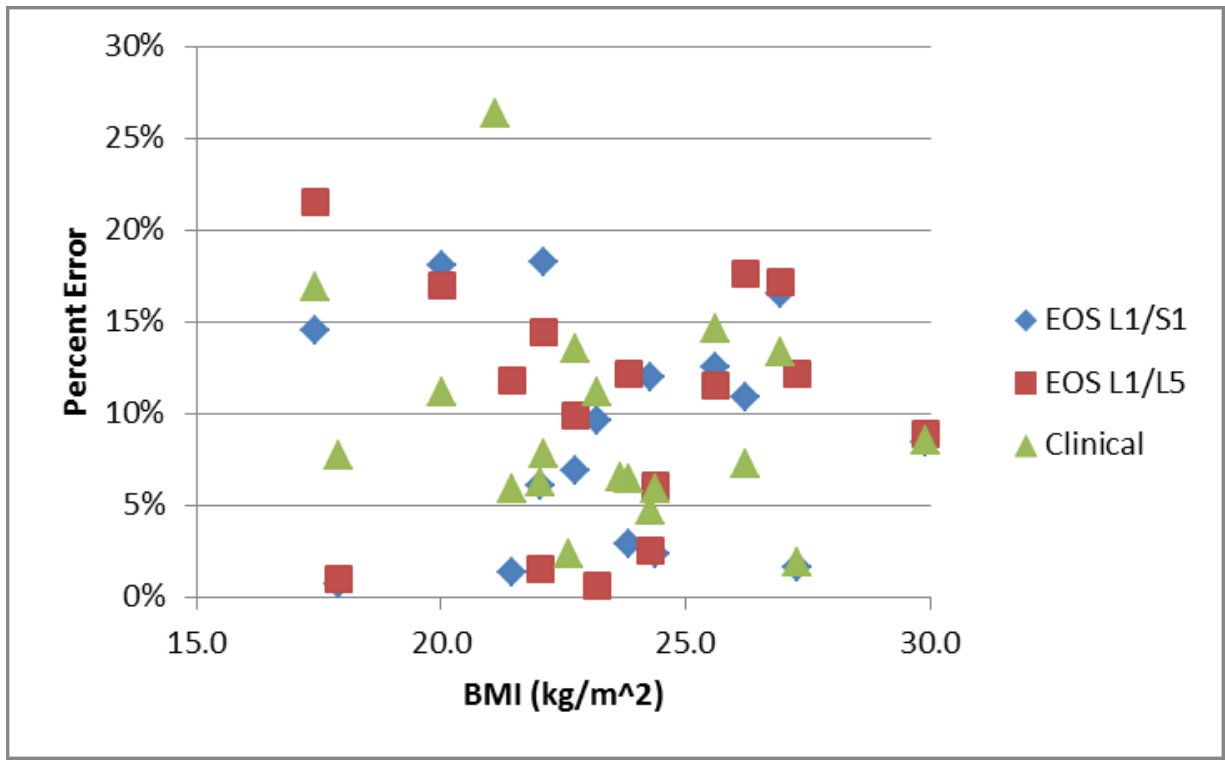

Figure B-2: Percent error vs BMI using the ratio method 


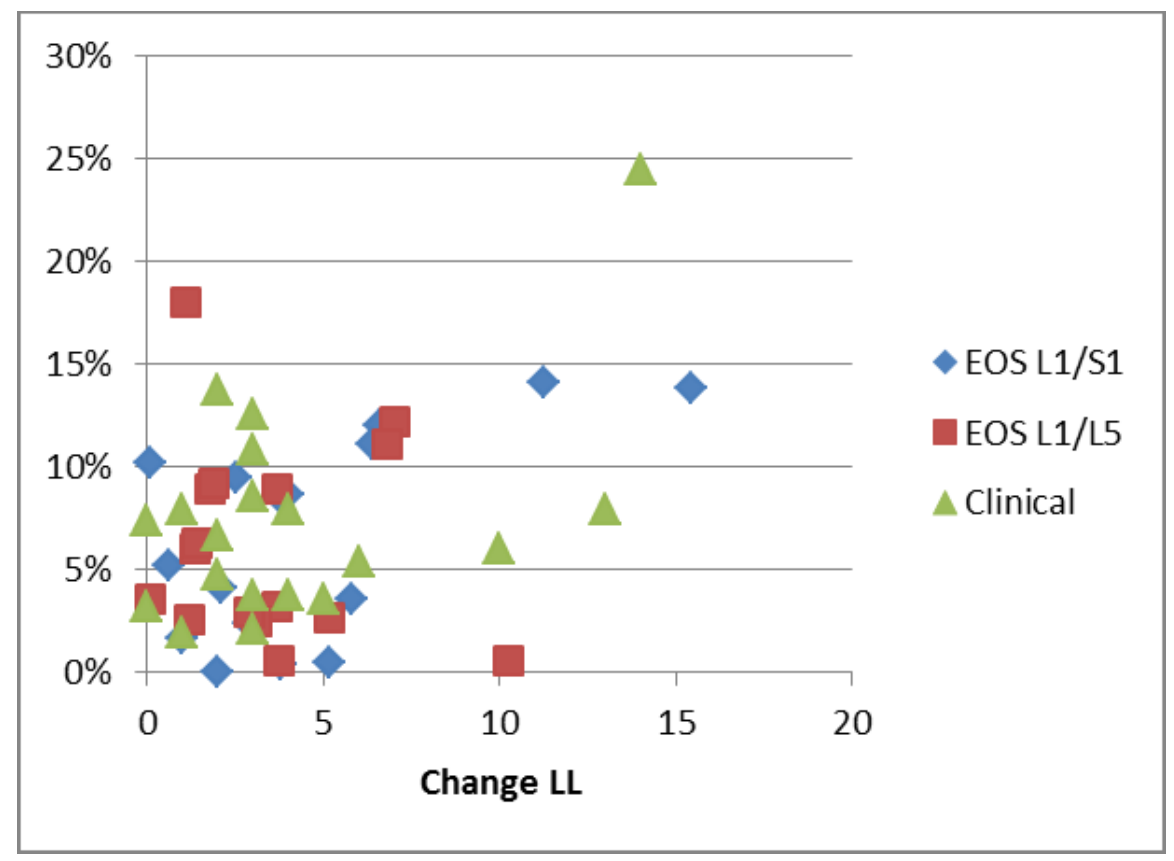

Figure B-3: Percent error vs change in anatomical LL using the difference method

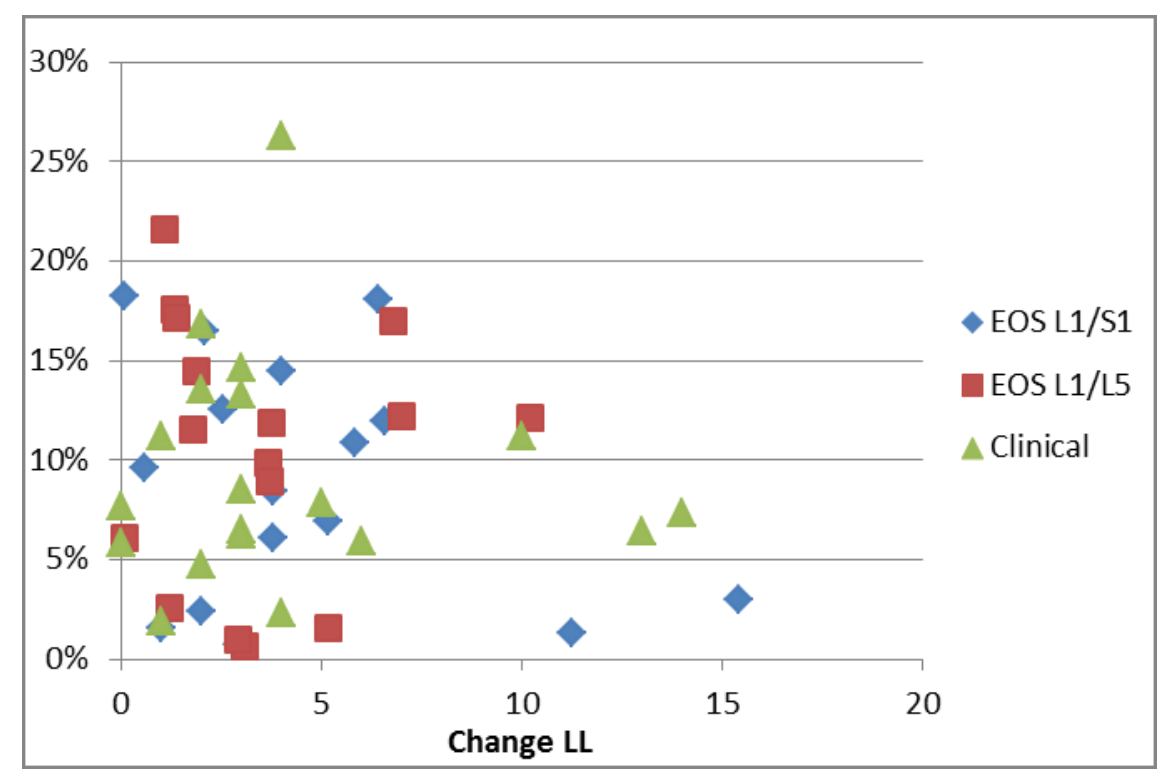

Figure B-4: Percent error vs change in anatomical LL using the ratio method 


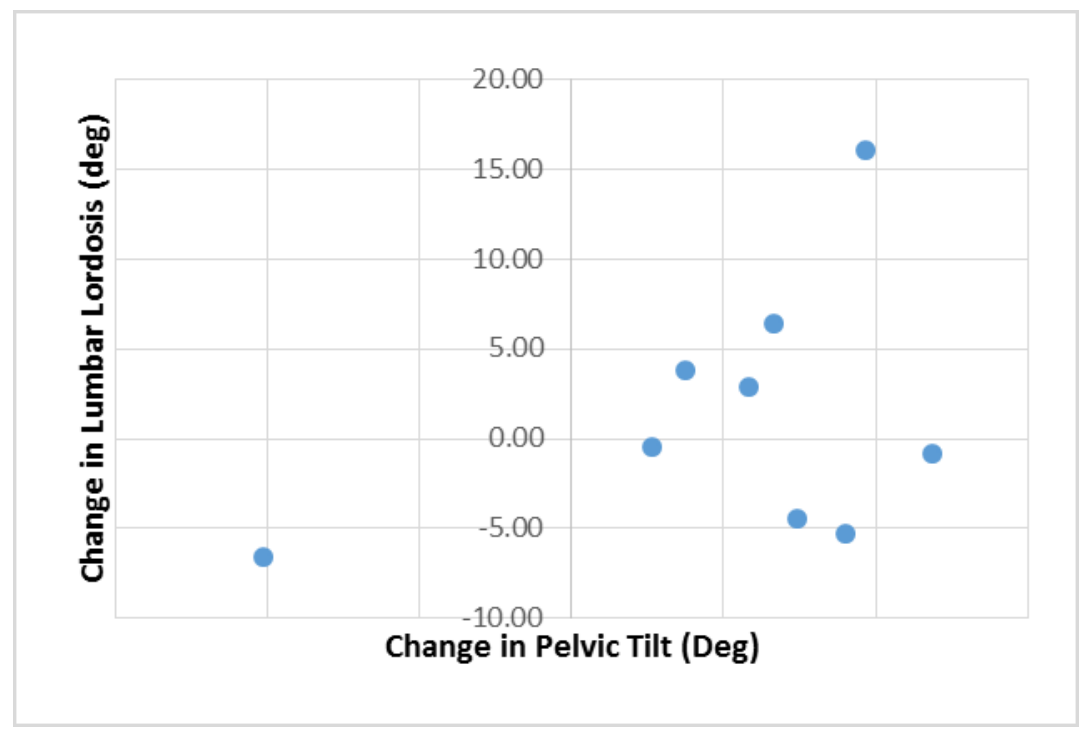

Figure B-5: Change in PT vs Change in LL at heal strike

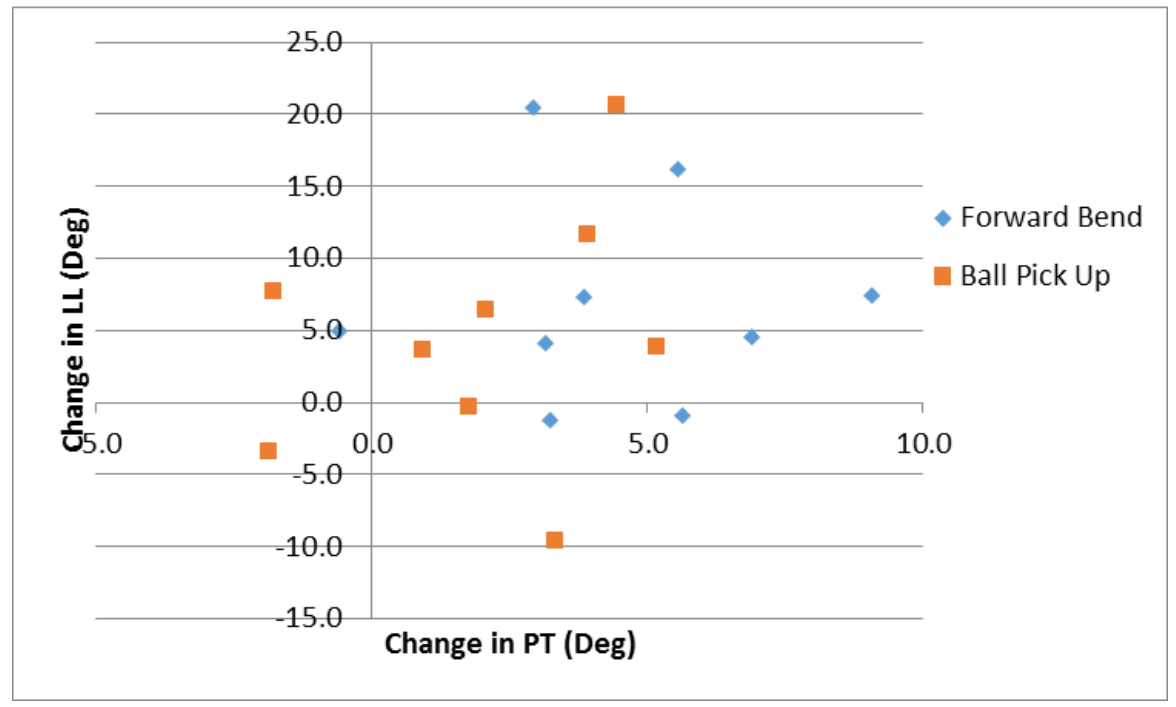

Figure B-6: Change in PT vs Change in LL while standing 


\section{VITA}

Michael Braman was born in Youngstown, Ohio in 1989. He attended the University of Dayton in Dayton, Ohio, where he earned a Bachelor's Degree in Chemical Engineering in 2010. After graduating he worked for Cargill, Inc. as a Manufacturing Engineer in Eddyville, Iowa. In 2013 he moved to Memphis, Tennessee to work as Process Development Engineer for Cargill, Inc. In 2014 Michael decided to pursue his Master's Degree in Biomedical Engineering. He worked under Dr. William Mihalko as a graduate research assistant in the Joint Program in Biomedical Engineering at the University of Tennessee Health Science Center and the University of Memphis. Michael graduated in 2016 with a Master's of Science Degree in Biomedical Engineering, focusing in biomechanics. 